\title{
An enhanced sequential fully implicit scheme for reservoir geomechanics
}

\author{
Omar Duran ${ }^{\mathrm{a}}$, Manouchehr Sanei ${ }^{\mathrm{b}}$, Philippe R. B. Devloo ${ }^{\mathrm{c}}$, Erick S.R. Santos ${ }^{\mathrm{d}}$ \\ ${ }^{a}$ Centre d'Enseignement et de Recherche en Mathématiques et Calcul scientifique, CERMICS (ENPC), Université \\ Paris-Est, 77455, Paris, France \\ ${ }^{b}$ Mechanical Engineering Department (FEM) - Petroleum Engineering Division, University of Campinas, \\ 13083-970, Campinas, SP, Brazil \\ ${ }^{c}$ Civil Engineering Department (FEC), University of Campinas, 13083-970, Campinas, SP, Brazil \\ ${ }^{d}$ Research and Development Center (Cenpes), Petrobras, Rio de Janeiro, RJ, Brazil
}

\begin{abstract}
In this paper, it is proposed an enhanced sequential fully implicit ESFI algorithm with a fixed stress split to approximate robustly poro-elastoplastic solutions related to reservoir geomechanics. The constitutive model considers the total strain effect on porosity/permeability variation and associative plasticity. The sequential fully implicit algorithm SFI is a popular solution to approximate solutions of a coupled system. Generally, the SFI consists of an outer loop to solve the coupled system, in which there are two inner iterative loops for each equation to implicitly solve the equations. The SFI algorithm occasionally suffers from slow convergence or even convergence failure. In order to improve the convergence (robustness) associated with SFI, a new nonlinear acceleration technique is proposed employing Shanks transformations in vector-valued variables to enhance the outer loop convergence, with a Quasi-Newton method considering the modified Thomas method for the internal loops. In this ESFI algorithm, the fluid flow formulation is defined by Darcy's law including nonlinear permeability based on Petunin model. The rock deformation includes a linear part being analyzed based on Biot's theory and a nonlinear part being established using Mohr-Coulomb associative plasticity for geomechanics. Temporal derivatives are approximated by an implicit Euler method and spatial discretizations are adopted using finite element in two different formulations. For the spatial discretization, two weak statements are obtained: the first one uses a continuous Galerkin for poro-elastoplastic and Darcy's flow; the second one uses a continuous Galerkin for poro-elastoplastic and a mixed finite element for Darcy's flow. Several numerical simulations are presented to evaluate the efficiency of ESFI algorithm in reducing the number of iterations. Distinct poromechanical problems in $1 \mathrm{D}, 2 \mathrm{D}$, and 3D are approximated with linear and nonlinear settings. Where appropriate, the results were verified with analytic solutions and approximated solutions with an explicit Runge-Kutta solver for 2D axisymmetric poroelastoplastic problems.
\end{abstract}

Keywords: Enhanced fixed-stress split iterative scheme, Poroelastoplasticity, Nonlinear acceleration, Mixed finite elements, Reservoir geomechanics

\section{Introduction}

The coupling of poro-elastoplastic rock deformation and fluid flow in porous media is significant in a diverse range of engineering fields, such as reservoir engineering, biomechanics, environmen-

Email addresses: omar.duran@enpc.fr (Omar Duran), manouchehr.sanei@gmail.com (Manouchehr Sanei), phil@fec.unicamp.br ( Philippe R. B. Devloo ), erick_slis@petrobras.com.br (Erick S.R. Santos) 
tal engineering, earthquake engineering, etc. [1. In petroleum reservoir engineering the coupling between fluid flow and deformation plays a crucial role in the production by reservoir compaction [2], ground land subsidence [3], caprock integrity [4], wellbore stability [5], and stress-dependent permeability of the reservoir rock [6]. Nonetheless, in conventional reservoir simulation, it has traditionally emphasized flow modeling and ignored mechanical deformation or simplified using fluid pressure-dependent rock compressibility relationships [7]. In order to consider the stress changes and deformation in reservoirs due to change in fluid pressure, the coupled fluid flow and mechanical deformation are required. Originally, the coupled flow and deformation was first developed under considerations of one-dimensional consolidation by Terzaghi [8]. Later, an extension of Terzaghi's theory to the third dimension was formulated by Biot [9]. In recent years, several researchers have been done in porous media to find approximated solutions for coupled fluid flow and deformation such as Settari and Mourits [10] which developed an iterative method for modular coupling of a reservoir simulator and a three-dimensional stress code. There, the authors have shown that the coupled approximation is significantly different in comparison with an uncoupled solution. In Yale et al. [11, remarkable differences were shown in the reservoir response during production. The conventional reservoir simulation and geomechanical modeling approaches miss the dynamic behavior between fluid pressure and rock deformation, while a fully coupled approach represents properly such a coupled effect. In Phillips and Wheeler [12] for the case of Biot's consolidation model of poroelasticity, a finite element procedure was reformulated for approximating the coupled fluid and mechanics. They approximate the pressure by a mixed finite element (MF) method and the displacements by a Continuous Galerkin (CG) method. In Dung [13], a fully coupled fluid flow and deformation modeling was developed to analyze the effect of reservoir compaction and subsidence on permeability and porosity variation. In Wei and Zhang [14], coupled fluid-flow and deformation modeling was developed for considering the effective stress effect on porosity/permeability changes in coal bed methane recovery. Recently there are rising interests in developing an application for the later; proper modeling of how porosity-permeability evolute is a key factor for modeling fluid flow through the rock masses [15, 16, 17].

An approximation for a poromechanic reservoir solution can normally fall into three categories: fully coupled (a monolithic approach), iteratively coupled (iterative sequential schemes), and loosely coupled. In the fully coupled scheme, the governing equations of flow and deformation are solved simultaneously at every time step. The fully coupled method requires careful implementation, sophisticated linear solvers, and preconditioners, and it is also computationally expensive [18, [19], [20]. In a loosely coupled scheme, the coupling between flow and deformation is solved only after a certain number of flow time steps [21]. The loosely coupled scheme is only conditionally stable and requires to estimate of when to update the deformation response. In the sequentially coupled scheme, several types of them are devised considering which variables are kept constant and the order for the sequential stages. For instance, by fixing the variation of total mean stress for reservoir equations and the fluid pressure for geomechanics, one possibility is to solve the flow problem first, and using the computed pressure approximation to solve the deformation problem. [22], [23], [24], [25]. The sequential method is iterated until the solution converges to the desired tolerance. This approach is more efficient than the fully coupled solution process either for linear and nonlinear poromechanics problems. Kim et al. [26], [27] proposed four types of sequential coupled procedures, such as drained split, undrained split, fixed strain split, and fixed stress split. Kim et al. 28] conclude that among sequential schemes the fixed stress split strategy is unconditionally stable and has better convergence properties. Later, Mikelić and Wheeler [29] demonstrated the convergence of the fixed stress split from a theoretical point of view using a contraction map with respect to an appropriately chosen metrics. The fixed stress split scheme consists of solving 
first the flow problem while freezing the mean total stress, and thus the mechanical part is solved using the values computed at the previous flow step. However, as nonlinearities are considered in the equations, the fixed stress split scheme can suffer from slow convergence or even convergence failure.

In Jiang and Tchelepi [30] the sequential fully implicit was extended with several nonlinear acceleration techniques to enhance the convergence and the stability of sequential schemes. The authors present an approach which extends the sequential fully implicit $(S F I)$ scheme with several nonlinear acceleration techniques to enhance the convergence and the stability of sequential schemes. They propose several acceleration techniques applied to the SFI in the context of the multiphase flow in porous media; Anderson acceleration, Quasi-Newton and Aitken's technique [31]. Recently, acceleration methods have been used to develop advanced numerical methods, such as domain decomposition [32, multigrid [33], fluid-structure interaction [34, thermomechanics [35]. The acceleration method of a single variable (scalar) was presented for the first time by Aitken [36]. Later, Aitken [31] applied the method for a multivariable function with interdependence between variables. Later, Plancq et al. [37] expressed several acceleration methods for vector sequences where most of them are generated based on the Aitken scalar acceleration algorithm. Jennings [38] presented two acceleration vector sequences named as first difference modulation $(F D M)$ or Aitken's transformation and second difference modulation $(S D M)$ or Anderson acceleration. In addition, in numerical analysis, transformations and extrapolations are mostly applied to slowly convergent sequences either from iterative processes or the partial summation of infinite series to decrease the number of function evaluations, such as Richardson extrapolation 39 and Shanks transformation [40], the latter being the focus for this contribution.

In this paper, the enhanced sequential fully implicit ESFI algorithm with the fixed stress split is proposed to approximate poro-elastoplastic problems considering nonlinearities as the total strain effect on porosity/permeability fields. In order to enhance the performance of SFI algorithm, two acceleration techniques including $F D M$ and $S D M$ are considered applying Shanks transformations as nonlinear acceleration for the external loop. For the internal loops, the modified Thomas method [67] is considered for both the reservoir and the geomechanics solver. The proposed nonlinear acceleration techniques are used for the first time within the context of $S F I$ for a poro-elastoplastic problem with variable permeability. In the ESFI algorithm, the fluid flow formulation is represented by a mass balance (single-phase) and Darcy's law consisting of nonlinear permeability Petunin model [41]. The poro-elastoplastic deformation includes a linear part being analyzed based on momentum balance and Biot's theory and a nonlinear part being established using an elastoplastic Mohr-Coulomb model [42]. Two numerical formulations are tested: continuous Galerkin for poro-elastoplastic and fluid flow; and continuous Galerkin for poroelastoplastic and mixed finite element approximation for fluid flow. The last one provides locally conservative flux approximations. In some selected cases the approximated solutions are compared with analytic or an in-house numerical solver based on an explicit Runge-Kutta approach. Numerical examples of poroelastoplastic in wellbore sector models are presented to evaluate the efficiency of the nonlinear acceleration techniques applied to the SFI algorithm. Among the acceleration methods, the proposed Shanks transformations provide good performance in terms of overall efficiency in a range of problems with certain nonlinearities. The implementation of the present study is done in the NeoPZ library [43], which is an object-oriented scientific computational environment, providing a framework for developing finite element schemes [44, 45, 46].

The structure for the manuscript is disposed of as follows. Firstly, the mathematical description and constitutive relationships are briefly presented, summarized, and discretized in space and time. Secondly, the linearization schemes used, the internal loop acceleration method and Shanks 
transformations for the external loop are provided. Thirdly, the numerical results are shown demonstrated the potential for the proposed enhanced sequential fully implicit scheme in 1D, 2D and 3D linear and nonlinear configurations. Finally, conclusions and discussions are provided.

\section{Mathematical Model}

The governing equations for coupled deformation and fluid flow are composed for a set of conservation laws and constitutive laws. The conservation equations are related to momentum and mass. At the macroscopic scale, two main approaches can be found depending on the Eulerian and Lagrangian descriptions: the pioneering research of Terzaghi and Biot [8, 47, 9, 48] provides a concise explanation for the Eulerian case; and the work of Coussy [49] develops the Lagrangian case. Under the quasi-static assumption the mechanical deformation can be expressed as follows (Rudnicki [50]):

$$
\operatorname{div}\left(\boldsymbol{\sigma}_{t}-\boldsymbol{\sigma}_{t}^{\circ}\right)=0
$$

where $\operatorname{div}(\cdot)\left[\mathrm{m}^{-1}\right]$ is the divergence operator on the deformed configuration, $\boldsymbol{\sigma}_{t}^{\circ}$ and $\boldsymbol{\sigma}_{t}[\mathrm{MPa}]$ are the initial and current total stress tensor. In terms of Cauchy effective stress tensor the momentum conservation equation becomes:

$$
\operatorname{div}\left(\boldsymbol{\sigma}-\boldsymbol{\sigma}^{\circ}-\alpha\left(p-p^{\circ}\right) \mathbf{I}\right)=0
$$

where $\boldsymbol{\sigma}^{\circ}$ and $\boldsymbol{\sigma}$ are the initial and current Cauchy effective stress tensor, $\alpha$ is the Biot's coefficient, $p^{\circ}$ and $p$ are the initial and current fluid pressure [MPa].

The set of equations are subjected to the corresponding initial ( referred with the superscript $\left.(\cdot)^{\circ}\right)$ and boundary conditions must be provided.

$$
\text { I.C. }=\left\{\begin{array}{ll}
p & p^{\circ} \text { on } \Omega \\
\mathbf{u}= & \mathbf{u}^{\circ} \text { on } \Omega
\end{array} \quad \text { B.C. }=\left\{\begin{array}{l}
\boldsymbol{\sigma}_{t} \mathbf{n}=\mathbf{t} \text { on } \partial \Omega_{\boldsymbol{\sigma}_{t}}^{N} \\
\mathbf{u}=\mathbf{u}_{D} \text { on } \partial \Omega_{\mathbf{u}}^{D}
\end{array}\right.\right.
$$

\subsection{Constitutive modeling for incremental elastoplastic deformation}

For a comprehensive description elastoplastic modeling the reader is referred to [42]. Only the concepts relevant to our work will be documented.

The nonlinear rock deformation is described by the theory of elastoplasticity for materials which present permanent deformations after some specific loading conditions. The total strain tensor $\boldsymbol{\epsilon}$ can be decomposed into two components as follows:

$$
\boldsymbol{\epsilon}=\boldsymbol{\epsilon}_{e}+\boldsymbol{\epsilon}_{p}
$$

Where $\boldsymbol{\epsilon}_{e}$ is the linear elastic component, and $\boldsymbol{\epsilon}_{p}$ is the non-linear plastic component. The superscript $(\dot{\xi})$ stands for an increment of the quantity $\xi$ in a pseudo time. Once the displacement increment $\dot{\mathbf{u}}$ is determined, the strain is defined by the strain-displacement relationship under the infinitesimal deformation assumption:

$$
\dot{\boldsymbol{\epsilon}}=\frac{1}{2}\left(\nabla \dot{\mathbf{u}}+\nabla^{T} \dot{\mathbf{u}}\right)
$$

And the effective stress increment $\dot{\boldsymbol{\sigma}}$ is determined by linear stress-strain relationship:

$$
\dot{\boldsymbol{\sigma}}=2 \mu \dot{\boldsymbol{\epsilon}}_{e}(\dot{\mathbf{u}})+\lambda \operatorname{tr}\left(\dot{\boldsymbol{\epsilon}}_{e}(\dot{\mathbf{u}})\right) \mathbf{I}
$$


where $\dot{\boldsymbol{\epsilon}}_{e}$ is the elastic strain increment. The parameters $\mu$ and $\lambda$ are the Lamé constants [MPa].

The elastic component is reversible and the plastic component represents a permanent deformation, and it is related to the history of irreversible deformations [51]. The elastoplastic deformation is a process that depends on the loading history. Thus, given an initial condition for $\mathbf{u}^{\circ}$ the elastoplastic history is composed of a series of loading steps, that define the variables associated to $\mathbf{u}$, like $\boldsymbol{\epsilon}$ and $\boldsymbol{\sigma}$ in an incremental fashion.

For the case of phenomenological constitutive approach, the material elastoplastic deformation can be explained by microstructural changes. These irreversible changes can be described by a set of macroscopic internal variables [52]:

$$
\chi_{k} ; k=1,2, \ldots
$$

where $\chi_{k}$ can be scalars, vectors, or other higher rank tensors. By regarding these internal variables, the Helmholtz free energy of the materials can be expressed as [53]:

$$
\mathbf{F}=\mathbf{F}\left(\epsilon_{e}, \chi_{k}\right)
$$

It can be decomposed as $\mathbf{F}^{e}$ elastic and $\mathbf{F}^{p}$ plastic portions. By differentiating Eq. (8) with respect to time and substituting the resulting expression into the Clausius-Duhem inequality, the following elastic constitutive relation is obtained by Hayakawa et al. [52]:

$$
\sigma=\bar{\rho} \frac{\partial \mathbf{F}_{e}}{\partial \boldsymbol{\epsilon}_{e}}
$$

The thermodynamic conjugate forces $\mathbf{A}_{k}$ are represented by:

$$
\mathbf{A}_{k}=\bar{\rho} \frac{\partial \mathbf{F}_{p}}{\partial \boldsymbol{\chi}_{k}}
$$

The elastoplastic deformation can be mathematically described by three fundamental axioms [42, 51]:

Yield criterion. Describes the plastic part through a yield function $\Phi=\Phi(\sigma, A)$, where $A=$ $\bar{\rho} \partial \mathbf{F} / \boldsymbol{\chi}$ is the hardening thermodynamic force and $\boldsymbol{\chi}$ is an internal damage variable. The yield function assumes negative values in an elastic part and null values in a plastic part.

Flow rule. Assumes the existence of a plastic potential function $\Psi=\Psi(\sigma, A)$, which specifies how the plastic deformation tensor $\epsilon_{p}$ evolves in the plasticity process $\dot{\epsilon}_{p}=\dot{\gamma} \mathbf{N}$, in which $\mathbf{N}(\sigma, A)=\partial \Psi / \partial \sigma$ is the flow direction, and $\dot{\gamma}$ is a plastic multiplier.

Hardening law. Specifies how the yield surface and internal damage variable $\dot{\chi}=\dot{\gamma} \mathbf{H}$ evolves in which, $\mathbf{H}(\sigma, A)=-\partial \Psi / \partial A$ is the hardening modulus.

Similarly to [50], the mass conservation for a slightly compressible fluid inside a saturated deformable material is expressed as:

$$
\frac{\partial(m)}{\partial t}+\operatorname{div}(\mathbf{q})=0
$$

where $m$ is the fluid content $\left[\mathrm{kg} \mathrm{m}^{-3}\right], \mathbf{q}=\rho_{f} \mathbf{v}_{f}$ is flux $\left[\mathrm{kg} \mathrm{s}^{-1} \mathrm{~m}^{-2}\right]$, and $\mathbf{v}_{f}\left[\mathrm{~ms}^{-1}\right]$ is the fluid velocity.

The corresponding initial and boundary conditions as follows: 


$$
\text { I.C. }=\left\{p=p^{\circ} \text { on } \Omega \quad \text { B.C. }=\left\{\begin{array}{l}
\mathbf{q} \cdot \mathbf{n}=q_{n} \text { on } \partial \Omega_{\mathbf{q}}^{N} \\
p=p_{D} \text { on } \partial \Omega_{p}^{D}
\end{array}\right.\right.
$$

2.2. Constitutive modeling for a slightly compressible fluid inside a deformable porous media

The fluid velocity is described by Darcy's law:

$$
\mathbf{v}_{f}=-\frac{\mathbf{K}}{\eta} \nabla p
$$

where $\mathbf{K}$ is the absolute permeability tensor $\left[\mathrm{m}^{2}\right], \eta$ is the dynamic fluid viscosity [Pas]. It is considered isotropic absolute permeability and it is expressed in terms of a scalar value $\kappa$ :

$$
\mathbf{K}=\kappa I
$$

During production, the effective stress can change because of the reduction of fluid pressure, or changes in other loading conditions. The way to simulate stress-dependent permeability is through an integrated constitutive model which is stress-strain dependent [54].

Permeability is controlled by the pore geometry and it is highly dependent on pore throat size and tortuosity. The reduction in porosity has a direct effect on absolute permeability [55]. Therefore, the relationship of permeability with the volumetric strain (alteration of porosity) leads to an implicit relation to stress-dependent permeability. A functional relationship for permeability only related to porosity is written as follows:

$$
\kappa=f(\phi)
$$

An empirical model which describes by the functional dependency 15 is expressed by Petunin et al. [41] as:

$$
\frac{\kappa}{\kappa^{\circ}}=\left(\frac{\phi}{\phi^{\circ}}\right)^{A}
$$

where $k^{\circ}$ and $k$ are the initial and current permeability. $A$ is the porosity exponent.

The total fluid content is expressed as:

$$
m=f(\mathbf{u}, p)=\phi \rho_{f}
$$

Equation (17) can be linearized using the expression for the Lagrangian porosity. The changes in porosity can be modeled by either the linearized poroelastic case [56] or the linearized poroelastoplastic case [27, 28, 49]. Without loss of generality the porosity change $\delta \phi=\phi-\phi^{\circ}$ can be expressed by a linear combination of two main effects: the deformation of the matrix and the deformation of the pores:

$$
\delta \phi=\delta \phi_{\text {matrix }}+\delta \phi_{\text {pore }}
$$

Introducing the pore compressibility as $S\left[\mathrm{MPa}^{-1}\right]$ :

$$
S=\left(\frac{(1-\alpha)\left(\alpha-\phi^{\circ}\right)}{K_{d r}}\right)
$$


where $\alpha$ is the Biot's coefficient, $\phi^{\circ}$ is the initial porosity, and $K_{d r}[\mathrm{MPa}]$ is the elastic rock bulk modulus in drained conditions.

The terms $\delta \phi_{\text {matrix }}$ and $\delta \phi_{\text {pore }}$ in expression (18) are functions of

- The volumetric elastic strain $\epsilon_{e v}=\epsilon_{v}-\epsilon_{p v}$;

- The plastic porosity $\phi_{p}$;

- The pore pressure.

The following expressions are suggested by Kim et al. [27], Coussy [49]:

$$
\begin{cases}\delta \phi_{\text {matrix }} & =\alpha\left(\epsilon_{e v}-\epsilon_{\text {ev }}^{\circ}\right)+\phi_{p}-\phi_{p}^{\circ} \\ \delta \phi_{\text {pore }} & =S\left(p-p^{\circ}\right)\end{cases}
$$

Combining equations 18,19 and 20 one obtains:

$$
\phi=\phi^{\circ}+\alpha\left(\epsilon_{e v}-\epsilon_{e v}^{\circ}\right)+\phi_{p}-\phi_{p}^{\circ}+\left(\frac{(1-\alpha)\left(\alpha-\phi^{\circ}\right)}{K_{d r}}\right)\left(p-p^{\circ}\right)
$$

It is generally difficult to evaluate the plastic porosity and in order to define it, a heuristic assumption is considered. A linear dependency of $\phi_{p}$ on $\epsilon_{p v}$ is written as:

$$
\phi_{p}=\alpha_{p} \epsilon_{p v}
$$

where the parameter $\alpha_{p}$ shares the same restrictions of $\alpha$, i.e. $\phi^{\circ} \leq \alpha_{p} \leq 1$ [49, 57]. This parameter is identified as the plastic effective stress coefficient [58, 57] or nonlinear Biot parameter [59].

There are experimental results that support the fact that $\alpha \neq \alpha_{p}$. Coussy [49] suggest that for a limestone under drained conditions $\alpha_{p} \approx 1$ while $\alpha=0.9$. For chalks Xie and Shao [60] have shown that $\alpha_{p} \approx 1$. However, other researchers Zhou et al. [58], Kim et al. [27, 28] assume that $\alpha=\alpha_{p}$. For simplicity $\alpha=\alpha_{p}$ is considered in this research.

The fluid density $\rho_{f}\left[\mathrm{~kg} \mathrm{~m}^{-3}\right]$ for a slightly compressible fluid is [61]:

$$
\rho_{f}=\rho_{f}^{\circ}\left(1+c_{f}\left(p-p^{\circ}\right)\right)
$$

Defining $S_{\epsilon}=\frac{1}{M}$ as the inverse of Biot's modulus $M[\mathrm{MPa}]$, it can be written as:

$$
S_{\epsilon}=S+\phi^{\circ} c_{f}
$$

Consequently, the linearized total fluid content can be expressed as in Kim et al. [27], Coussy [49], da Silva et al. [59]:

$$
\phi \rho_{f}=\rho_{f}^{\circ}\left(\phi^{\circ}+\alpha\left(\epsilon_{e v}-\epsilon_{e v}^{\circ}\right)+\phi_{p}-\phi_{p}^{\circ}+S_{\epsilon}\left(p-p^{\circ}\right)\right)
$$

The volumetric strain $\epsilon_{e v}$ can be related to volumetric total stress $\sigma_{t v}=\operatorname{tr}\left(\boldsymbol{\sigma}_{t}\right) / 3$ as follows:

$$
\left(\sigma_{t v}-\sigma_{t v}^{\circ}\right)+\alpha\left(p-p^{\circ}\right)=K_{d r}\left(\epsilon_{e v}-\epsilon_{e v}^{\circ}\right)
$$

Following the ideas in Kim et al. [62], the elastoplastic tangent bulk modulus $K_{d r_{e p}}$ can be related to the mean effective stress variation $\delta \sigma_{v}=\operatorname{tr}(\delta \boldsymbol{\sigma}) / 3$, considering that $\delta \sigma_{v}=\delta \sigma_{t v}+\alpha \delta p$ : 


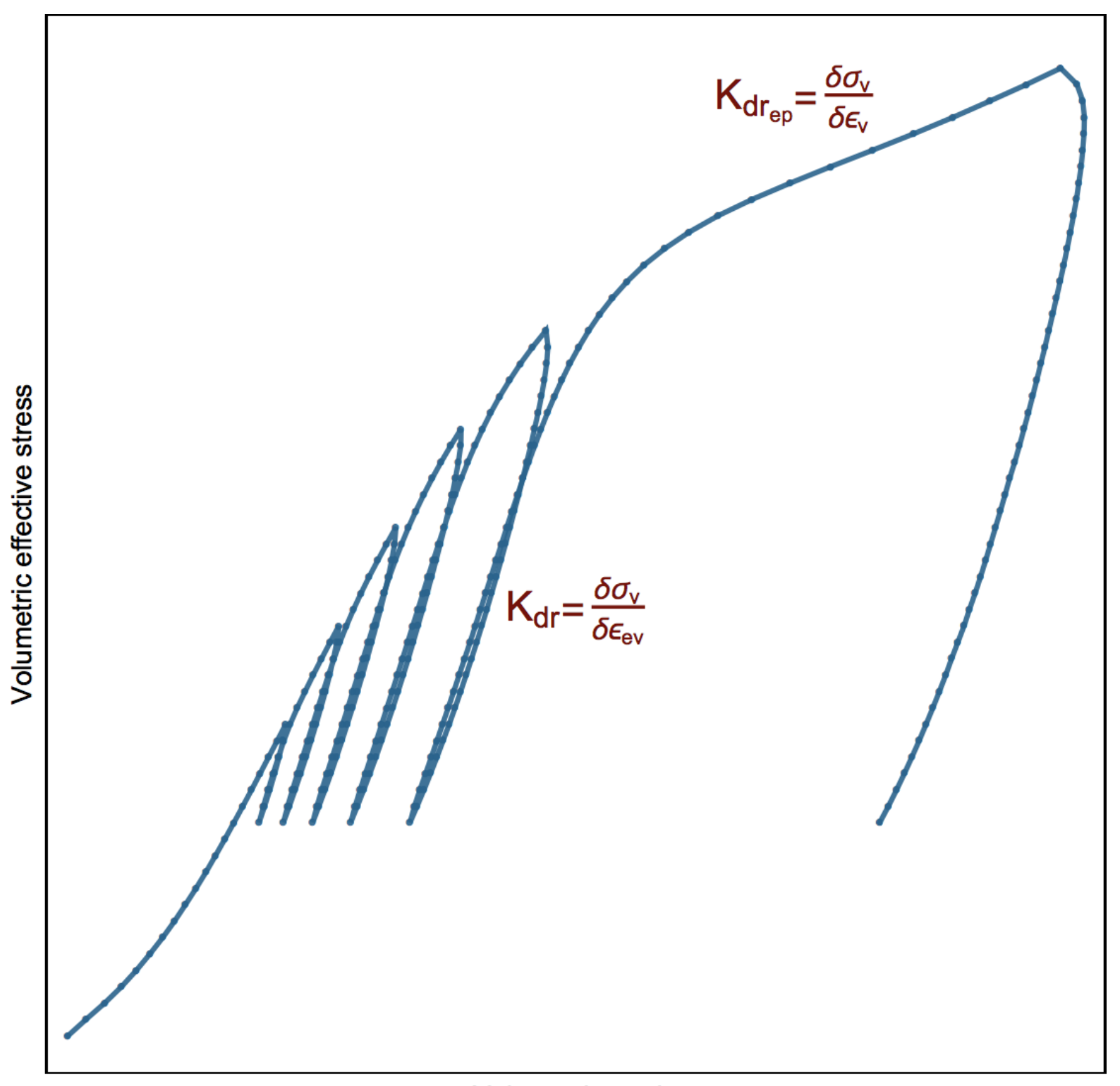

Volumetric strain

Figure 1: Schematic hydrostatic test.

$$
\delta \sigma_{v}=K_{d r_{e p}} \delta \epsilon_{v}
$$

The figure 1 shows a schematic hydrostatic test and how the material data $K_{d r}$ and $K_{d r_{e p}}$ are defined in terms of volumetric stress variation, volumetric strain, and volumetric elastic strain. Kim et al. 62 state that Biot parameter $\alpha$ is related to the elastoplastic tangent bulk modulus $K_{d r_{e p}}$ as follows:

$$
\alpha=1-\frac{K_{d r_{e p}}}{K_{s}}
$$

The expressions (27) and (28), are used to update $\alpha$.

By inserting equation (26) into equation (25), an alternative expression for total fluid content is obtained as documented in Kim et al. [62]: 


$$
\phi \rho_{f}=\rho_{f}^{\circ}\left(\phi^{\circ}+\frac{\alpha}{K_{d r}}\left(\sigma_{t v}-\sigma_{t v}^{\circ}\right)+\phi_{p}-\phi_{p}^{\circ}+\left(S_{\epsilon}+\frac{\alpha^{2}}{K_{d r}}\right)\left(p-p^{\circ}\right)\right)
$$

where $\sigma_{t v}^{\circ}$ and $\sigma_{t v}$ are the initial and current volumetric total stress [MPa].

In the same way, by inserting equation (26) into equation (20), an alternative expression for $\delta \phi_{\text {matrix }}$ is obtained:

$$
\delta \phi_{\text {matrix }}=\frac{\alpha}{K_{d r}}\left(\sigma_{t v}-\sigma_{t v}^{\circ}\right)+\frac{\alpha^{2}}{K_{d r}}\left(p-p^{\circ}\right)+\phi_{p}-\phi_{p}^{\circ}
$$

Finally, using the expression $(29)$ as a constitutive relationship, the mass balance equation can be rewritten as:

$$
\frac{\partial\left(\phi \rho_{f}\right)}{\partial t}+\operatorname{div}(\mathbf{q})=0
$$

\subsection{Summary for the mathematical model}

The strong form for the poroelastic equations is summarized in two sets of equations, one for the mathematical description for poro-elastoplastic and another for Darcy's flow, in tables 1 and 2 , respectively.

\begin{tabular}{|c|c|}
\hline \multicolumn{2}{|c|}{ Conservation law } \\
\hline \hline \multicolumn{2}{|c|}{ Constitutive laws } \\
\hline Momentum & $\operatorname{div}\left(\boldsymbol{\sigma}-\boldsymbol{\sigma}^{\circ}-\alpha\left(p-p^{\circ}\right) \mathbf{I}\right)=0$ \\
\hline Cauchy effective stress tensor & $\boldsymbol{\sigma}=2 \mu \boldsymbol{\epsilon}_{e}(\mathbf{u})+\lambda \operatorname{tr}\left(\boldsymbol{\epsilon}_{e}(\mathbf{u})\right) \mathbf{I}$ \\
\hline Total strain & $\boldsymbol{\epsilon}=\boldsymbol{\epsilon}_{e}+\boldsymbol{\epsilon}_{p}$ \\
\hline Total volumetric strain & $\epsilon_{v}=\epsilon_{e v}+\epsilon_{p v}$ \\
\hline
\end{tabular}

Table 1: Equations for poroelastoplasticity.

\begin{tabular}{|c|c|}
\hline \multicolumn{2}{|c|}{ Conservation law } \\
\hline \hline Mass & $\frac{\partial\left(\phi \rho_{f}\right)}{\partial t}+\operatorname{div}(\mathbf{q})=0$ \\
\hline Darcy's law & $\mathbf{q}=-\rho_{f} \frac{\kappa(\phi) I}{\eta} \nabla p$ \\
\hline Lagrangian porosity & $\phi=\phi^{\circ}+\alpha\left(\epsilon_{e v}-\epsilon_{e v}^{\circ}\right)+\phi_{p}-\phi_{p}^{\circ}+S\left(p-p^{\circ}\right)$ \\
\hline Fluid content & $\frac{\phi \rho_{f}}{\rho_{f}^{\circ}}=\phi^{\circ}+\frac{\alpha}{K_{d r}}\left(\sigma_{t v}-\sigma_{t v}^{\circ}\right)+\phi_{p}-\phi_{p}^{\circ}$ \\
& $+\left(S_{\epsilon}+\frac{\alpha^{2}}{K_{d r}}\right)\left(p-p^{\circ}\right)$ \\
\hline Permeability model & $\frac{\kappa}{\kappa^{\circ}}=\left(\frac{\phi}{\phi^{\circ}}\right)^{A}$ \\
\hline
\end{tabular}

Table 2: Equations for Darcy's flow.

The strong form is composed of equations in tables 1 and 2, Naturally, the system is fully coupled and it is completed by applying the Dirichlet and Neumann boundary conditions as documented by the equations (3) and (12). 


\subsection{Spatial and temporal discretization}

The temporal derivatives are approximated by the implicit Euler method. The index $\left.(\cdot)\right|^{n-1}$ stands for terms evaluated at the last state $n-1$, and the index $\left.(\cdot)\right|^{n}$ stands for terms evaluated at the current state $n$. In spatial variables, a finite element discretization is adopted. The second-order derivatives of conservation laws presented in tables 1 and 2 are reduced to first-order derivatives by the use of Gauss divergence theorem. Two possible numerical approximations are considered:

1. $\mathrm{H}^{1}$ approximations are used for modeling poro-elastoplasticity and Darcy's flow;

2. $\mathrm{H}^{1}$ approximations are used for modeling poro-elastoplasticity and a mixed finite element method using $\mathrm{H}$ (div) approximations for the Darcy's flow.

Recently, the second approach has received interest for its higher precision in terms of fluid flux Li et al. [19, Wheeler and Gai [24], da Silva et al. [59], Both et al. [63].

Before introducing the spatial discretization, consider a partition $\mathcal{T}_{\mathrm{H}}=\left\{\Omega_{e}\right\}$ of the domain $\Omega$ by convex elements. The boundary of each element is denoted $\partial \Omega_{e}$ and its external unit normal vector $\mathbf{n}$. The symbol $H$ indicates the maximum diameter of the elements $\Omega_{e}$ and the notation $d$ stands for problem dimension. In brief, the inner products that come from the Galerkin's method are expressed as $(\cdot, \cdot)_{\Omega_{e}}$ for volumetric integrals and $(\cdot, \cdot)_{\partial \Omega_{D / N}}$ for boundary integrals with Dirichlet or Neumann data. The strong form is written in the continuous case and then converted to its discrete version by using a finite-dimensional functional approximation spaces. The first step is avoided for briefness and the expressions are provided just for the discrete forms.

\subsubsection{Discrete Weak statement I}

In this weak statement, the displacement field $\mathbf{u}_{h}$ is used as a state variable for the poroelastoplastic problem and the pressure field $p_{h}$ is used for Darcy's flow problem. The following functional approximation space is required for vector fields:

$$
\mathrm{H}^{1}(\Omega)^{d}=\left\{v \in L^{2}(\Omega) \mid \nabla v \in\left[\mathrm{L}^{2}(\Omega)\right]^{d}\right\}
$$

and for scalar fields:

$$
\mathrm{H}^{1}(\Omega)=\left\{v \in L^{2}(\Omega) \mid \nabla v \in \mathrm{L}^{2}(\Omega)\right\}
$$

The finite-dimensional subspace $\mathbf{V}_{h} \subset \mathbf{V} \subset \mathrm{H}^{1}(\Omega)^{d}$ is used for approximating continuous vector fields:

$$
\mathbf{V}_{h}=\left\{\mathbf{v} \in \mathrm{H}^{1}(\Omega)^{d} \mid \mathbf{v}=0 \text { on } \partial \Omega_{D}\right\} \text { and } \mathbf{V}_{h} \subset \mathbf{V}
$$

The finite-dimensional subspace $U_{h} \subset U \subset \mathrm{H}^{1}(\Omega)$ is used for approximating continuous scalar fields :

$$
U_{h}=\left\{u \in \mathrm{H}^{1}(\Omega) \mid u=0 \text { on } \partial \Omega_{D}\right\} \text { and } U_{h} \subset U
$$

The approximation space $\mathbf{V}_{h}$ and $U_{h}$, respectively are used for displacements $\mathbf{u}_{h}$ and pressure $p_{h}$. Using admissible members of $\mathbf{V}_{h}$ and $U_{h}$ as a test and trial functions in the sense of Galerkin's method, the discrete formulation is expressed as the solution of two subproblems:

For poroelastoplasticity. Given the prescribed field $p_{h}^{n} \in U_{h}$ and the initial data, find $\mathbf{u}_{h}^{n} \in \mathbf{V}_{h}$ such that: 


$$
\sum_{\Omega_{e} \in \mathcal{T}}\left(\boldsymbol{\sigma}_{h}{ }^{n}-\boldsymbol{\sigma}_{h}^{\circ}-\alpha\left(p_{h}^{n}-p_{h}^{\circ}\right) \mathbf{I}, \boldsymbol{\epsilon}\left(\boldsymbol{\phi}_{u}\right)\right)_{\Omega_{e}}-\sum_{\Omega_{e} \in \mathcal{T}}\left(\mathbf{t}^{n}-\mathbf{t}^{\circ}, \boldsymbol{\phi}_{u}\right)_{\partial \Omega_{N}}=0
$$

holds for all $\phi_{u} \in \mathbf{V}_{h}$.

The expression above is completed with the corresponding boundary conditions (3).

For Darcy's flow. Given the prescribed field $\mathbf{u}_{h}^{n} \in \mathbf{V}_{h}$, the last state and the initial data, find $p_{h}^{n} \in U_{h}$ such that:

$$
\begin{aligned}
\sum_{\Omega_{e} \in \mathcal{T}}\left(\rho_{f}^{n} \frac{\kappa^{n} \mathbf{I}}{\eta} \nabla p_{h}^{n}, \nabla \phi_{p}\right)_{\Omega_{e}}-\sum_{\Omega_{e} \in \mathcal{T}}\left(q_{n}, \phi_{p}\right)_{\partial \Omega_{N}} & \\
& +\sum_{\Omega_{e} \in \mathcal{T}}\left(\frac{\left.\phi \rho_{f}\right|^{n}-\left.\phi \rho_{f}\right|^{n-1}}{\Delta t}, \phi_{p}\right)_{\Omega_{e}}=0
\end{aligned}
$$

holds for all $\phi_{p} \in U_{h}$.

The expression above is completed with the corresponding boundary conditions $(12)$.

\subsubsection{Discrete Weak statement II}

In this weak statement, as the first form the displacement field $\mathbf{u}_{h}$ is used as the state variable for the poroelastoplastic problem, but two fields, the flux $\mathbf{q}_{h}$ and pressure $p_{h}$ are used as state variables for the Darcy's flow problem. Additional approximation spaces are required for the variables $p_{h} \in W=L^{2}(\Omega)$ and $\mathbf{q}_{h} \in \mathrm{H}(\operatorname{div}, \Omega)$, where:

$$
\mathrm{H}(\operatorname{div}, \Omega)=\left\{\mathbf{w} \in\left[\mathrm{L}^{2}(\Omega)\right]^{d} \mid \operatorname{div}(\mathbf{w}) \in \mathrm{L}^{2}(\Omega)\right\}
$$

And the corresponding function space $\mathbf{Q}$ and finite subspace $\mathbf{Q}_{h}$ for $\mathrm{H}(\operatorname{div}, \Omega)$ :

$$
\mathbf{Q}=\left\{\mathbf{q} \in \mathrm{H}(\operatorname{div}, \Omega) \mid \mathbf{q} \cdot \mathbf{n}=0 \text { on } \partial \Omega_{N}\right\} \text { and } \mathbf{Q}_{h} \subset \mathbf{Q}
$$

The approximation subspace $\mathbf{V}_{h}$ is used for $\mathbf{u}_{h}$. Using admissible members of $\mathbf{Q}_{h} \times L^{2}(\Omega)$ as a test and trial functions in the sense of Galerkin's method. The discrete formulation can be expressed as follows.

For poro-elastoplasticity: identical to the case provided in 2.4.1.

For Darcy's flow. The problem is expressed in a mixed form [64], as:

Given the prescribed field $\mathbf{u}_{h}^{n} \in \mathbf{V}_{h}$, the last state and the initial data, find $\left(\mathbf{q}_{h}, p_{h}\right)^{n} \in \mathbf{Q}_{h} \times$ $L^{2}(\Omega)$ such that:

$$
\begin{array}{cc}
\sum_{\Omega_{e} \in \mathcal{T}}\left(\left(\rho_{f}^{n} \frac{\kappa^{n} \mathbf{I}}{\eta}\right)^{-1} \mathbf{q}_{h}^{n}, \boldsymbol{\phi}_{q}\right)_{\Omega_{e}}+\sum_{\Omega_{e} \in \mathcal{T}}\left(p_{h}^{n}, \boldsymbol{\phi}_{q} \cdot \mathbf{n}\right)_{\partial \Omega_{D}} & \\
-\sum_{\Omega_{e} \in \mathcal{T}}\left(p^{n}, \operatorname{div}\left(\phi_{q h}\right)\right)_{\Omega_{e}} & =0 \\
\sum_{\Omega_{e} \in \mathcal{T}}\left(\operatorname{div}\left(\mathbf{q}_{h}^{n}\right), \phi_{p}\right)_{\Omega_{e}}+\sum_{\Omega_{e} \in \mathcal{T}}\left(\frac{\left.\phi \rho_{f}\right|^{n}-\left.\phi \rho_{f}\right|^{n-1}}{\delta t}, \phi_{p}\right)_{\Omega_{e}} & =0
\end{array}
$$

holds for all $\left(\phi_{q}, \phi_{p}\right) \in \mathbf{Q}_{h} \times L^{2}(\Omega)$.

The expression above is completed with the corresponding boundary conditions $(12)$. 


\section{Linearization schemes}

The most classical and straight forward linearization scheme corresponds to the fully coupled monolithic solver, which is intrinsically expensive for either the weak statements detailed in subsections 2.4.1 or 2.4.2. For the case of the monolithic solver applied to the weak statement 2.4.1. there are iterative methods that can be applied to obtain cost-effective approximations for the monophase Castelletto et al. [65], and multiphase White et al. [66] cases. However, these approximations do not provide a locally conservative approximation for the fluid flux. On the other hand, the monolithic solver applied to weak statement 2.4 .2 provides a precise locally conservative flux approximations but is not recommended, because the linear solver needs to decompose non-symmetric system of equations with saddle-point structure as a consequence of the mixed formulation.

Sequential schemes provide the possibility of breaking the solution of the coupled problem into subproblems. Their efficiency depends on the development of efficient split operators and efficient solvers for each subproblem. In consequence, the weak statement 2.4 .2 can be handled efficiently by the use of an appropriated sequential scheme. Hence, the section is dedicated for describing several ingredients and the ideas behind the construction for the proposed enhanced sequential fully implicit algorithm. The provided descriptions are:

(i) The notation adopted for the residual expressions;

(ii) A monolithic solver based on a classical Newton method used to verify the correctness of the implementations;

(iii) A concise description of several sequential split operators;

(i) The description for the selected fixed stress split operator;

(ii) The ideas behind the sequential fully implicit algorithm with a fixed stress split. It can be seen as a sequential scheme based on physical principles that approximates the nonlinear solution through a series of nonlinear approximations;

(iii) Information transfer between modules. It represents the manner of how the information is transferred between the separate modules;

(iv) Nonlinear acceleration techniques;

(i) The accelerated convergence for the internal loops. The internal nonlinear solvers are Quasi-Newton methods with extra evaluation for the residual expressions;

(ii) Nonlinear acceleration for external loop. The acceleration is provided by a novel Shankslike augmented acceleration for $F D M / S D M$ transformations;

(v) Enhanced SFI algorithm.

The purpose of the first three descriptions is to provide the fundamental ideas about the sequential fully implicit algorithm SFI. It is important to point out $S F I$ is performed as a sequence of two internal loops nested into a third external loop.

The main contribution is to introduce the main ideas for the acceleration of the internal loops, as well as new Shanks augmented acceleration considering the sequence transformations FDM and $S D M$ for the external loop [38]. Finally, the Enhanced SFI algorithm ESFI is composed applying internal and external nonlinear accelerations techniques over the standard SFI algorithm. Some implementations details are given through the subsection and the accelerated convergence for the proposed ESFI is presented later in several numerical examples. 


\subsection{Notation adopted for residuals expressions}

The incremental form of the weak statements introduced above lead to the definition of the following residual expressions:

For discrete weak statement 2.4.1,

$$
\begin{aligned}
& r_{u}\left(\mathbf{u}_{h}, p_{h}\right)^{n}=\sum_{\Omega_{e} \in \mathcal{T}}\left(\boldsymbol{\sigma}_{h}^{n}-\boldsymbol{\sigma}_{h}^{\circ}-\alpha\left(p_{h}^{n}-p_{h}^{\circ}\right) \mathbf{I}, \boldsymbol{\epsilon}\left(\boldsymbol{\phi}_{u}\right)\right)_{\Omega_{e}} \\
& \sum_{\Omega_{e} \in \mathcal{T}}\left(\mathbf{t}^{n}-\mathbf{t}^{\circ}, \boldsymbol{\phi}_{u}\right)_{\partial \Omega_{N}} \\
& r_{p}\left(p_{h}, \mathbf{u}_{h}\right)^{n}=\left.\sum_{\Omega_{e} \in \mathcal{T}}\left(\rho_{f}^{n} \frac{\kappa^{n} \mathbf{I}}{\eta} \nabla p_{h}^{n}, \nabla \phi_{p}\right)_{\Omega_{e}}\right|^{n-1} \\
&-\sum_{\Omega_{e} \in \mathcal{T}}\left(q_{h}^{n}, \phi_{p}\right)_{\partial \Omega_{N}}+\sum_{\Omega_{e} \in \mathcal{T}}\left(\frac{\left.\phi \rho_{f}\right|^{n}-\left.\phi \rho_{f}\right|^{n}}{\delta t}, \phi_{p}\right)_{\Omega_{e}}
\end{aligned}
$$

For discrete weak statement 2.4.2.

$$
r_{p}\left(\mathbf{q}_{h}, p_{h}, \mathbf{u}_{h}\right)^{n}=\left\{\begin{array}{l}
\sum_{\Omega_{e} \in \mathcal{T}}\left(\left(\rho_{f}^{n} \frac{\kappa^{n} \mathbf{I}}{\eta}\right)^{-1} \mathbf{q}_{h}^{n}, \boldsymbol{\phi}_{q}\right)_{\Omega_{e}}\left(\sum_{\Omega_{e} \in \mathcal{T}}\left(p_{h}^{n}, \boldsymbol{\phi}_{q} \cdot \mathbf{n}\right)_{\partial \Omega_{D}}-\sum_{\Omega_{e} \in \mathcal{T}}\left(p^{n}, \operatorname{div}\left(\phi_{q h}\right)\right)_{\Omega_{e}} ;\right. \\
\sum_{\Omega_{e} \in \mathcal{T}}\left(\operatorname{div}\left(\mathbf{q}_{h}^{n}\right), \phi_{p}\right)_{\Omega_{e}}+\sum_{\Omega_{e} \in \mathcal{T}}\left(\frac{\left.\phi \rho_{f}\right|^{n}-\left.\phi \rho_{f}\right|^{n-1}}{\delta t}, \phi_{p}\right)_{\Omega_{e}}
\end{array}\right.
$$

For simplicity, all the explanations provided in this section are associated with the weak statement 2.4.2. Given the data at state $\left(\mathbf{q}_{h}, p_{h}, \mathbf{u}_{h}\right)^{n-1} \in \mathbf{Q}_{h} \times L^{2}(\Omega) \times \mathbf{V}_{h}$, the residuals are approximated at some state $\left(\mathbf{q}_{h}, p_{h}, \mathbf{u}_{h}\right)^{n} \in \mathbf{Q}_{h} \times L^{2}(\Omega) \times \mathbf{V}_{h}$.

In brief, the residuals expressions associated with an iteration index $i$ and it can be denoted as :

$$
\begin{aligned}
& \left.r_{u}^{i}\right|^{n}:=\quad r_{u}\left(\mathbf{u}_{h}^{i}, p_{h}^{i}\right)^{n} \\
& \left.r_{p}^{i}\right|^{n}:= \begin{cases}r_{p}\left(\mathbf{p}_{\mathbf{h}}, \mathbf{u}_{h}\right)^{n} & \text { Discrete weak statement I } \\
r_{p}\left(\mathbf{q}_{h}, p_{h}, \mathbf{u}_{h}\right)^{n} & \text { Discrete weak statement II }\end{cases}
\end{aligned}
$$

\subsection{A monolithic solver}

A classical monolithic solver is obtained by linearizing the residuals expressions $\left.r_{u}^{i}\right|^{n}$ and $\left.r_{p}^{i}\right|^{n}$, computing the consistent tangent matrices and solving the equations simultaneously. In this research, the monolithic solver is applied to the residuals $\left.r_{u}^{i}\right|^{n}$ and $\left.r_{p}^{i}\right|^{n}$ defined by the weak statement 2.4 .1 .

The fully coupled solver is computationally expensive. It is used in this publication for comparison purpose only.

\subsection{Fixed stress split as a sequential fully implicit algorithm}

A sequential scheme can provide a cost-effective approximated solution for the fully coupled problem. From the implementation point of view, sequential schemes have the natural advantage of modular programming and facilitate the use of specialized numerical methods: one to solve the fluid flow and a different scheme for converging the mechanical deformation problems [10, 24, 26, 30]. 
Kim et al. [26, 27] document four types of sequential procedures, including Drained split, Undrained split, Fixed-strain split, and Fixed-stress split. The last two procedures were originally proposed by Settari and Mourits [10].

The different procedures are related to the fluid content (25) or (29) presented in the temporal derivate of the expression (31). For the sake of clarity, the subscript $(\xi)_{h}$ is removed from the discrete variables. A concise description of their physical meaning is provided as follows:

Drained: In this scheme, the temporal variation of the quantity $p$ is ignored when solving the solid mechanics problem, i.e. $\delta p=0$. The split is useful when the coupling between fluid flow and rock deformation is weak or decoupled, i.e. the pressure changes slowly in relation to the deformation process.

Undrained: In this scheme, the temporal variation of the quantity $\phi \rho_{f}$ is neglected, i.e. $\delta \phi \rho_{f}=0$. This split is suitable to approximate the undrained response of poroelastic bodies surrounded by impervious boundaries, e.g. the initial condition of a closed reservoir; initial total stress and fluid pressure must be in equilibrium.

Fixed-strain: In this scheme, the fluid mass content is expressed as a function of pressure and total strain as in equation (25). The temporal variation of total volumetric strain $\epsilon_{v}$ is kept constant, i.e. $\delta \epsilon_{v}=0$. The split is employed when the pressure changes rapidly in relation to the deformation process, e.g. the pressure changes due to reservoir depletion.

Fixed-stress: In this scheme, the fluid mass content is expressed as a function of pressure and total stress as in equation (29). The temporal variation of mean total stress $\sigma_{t v}$ is frozen, i.e. $\delta \sigma_{t v}=0$. The split introduces an extra compressibility term for the pressure variable, making the temporal scales for $p$ and $\sigma_{t v}$ more separated in relation to the Fixed-strain case. It is useful when the pressure changes rapidly in relation to the deformation process, e.g. the pressure changes due to a producer well.

Kim et al. [28] state that the Fixed-stress split strategy is unconditionally stable and has better convergence properties compared to the other schemes. The better performance of the fixed stress scheme is because the variation of the total stress is slow compared with the other variables. Also, Mikelić and Wheeler [29] demonstrated that the convergence of the fixed stress split scheme using a contraction map with respect to an appropriately chosen metric.

Several other authors explain and use the Fixed-stress split sequential scheme as documented in [10, 28, 29, 59]. For this reason, the Fixed-stress split scheme is chosen as the sequentially coupled scheme in this work.

\subsection{A Fixed-stress split for poro-elastoplastic (FSS)}

Regarding the expressions (20) for porosity changes, the implementation for the Fixed Stress split can be established utilizing two main stages:

1. The approximation for the reservoir pressure considering implicitly $\delta \phi_{\text {pore }}$ and explicitly $\delta \phi_{\text {matrix }}$

2. The approximation of the geomechanics response considering implicitly $\delta \phi_{\text {matrix }}$.

As in [10, 29] an auxiliary variable called porosity correction $\delta \phi^{*}$ is introduced to perform the coupling between the subproblems. This variable accounts for the implicit porosity correction of the geomechanic response. The figure 2 shows how the stages are performed during a given time 
step $\Delta t$. Starting with the external iteration index $m \rightarrow 1$, the first step is to set $\delta \phi^{* m-1} \rightarrow 0$ and to add $\frac{\left(\alpha^{n-1}\right)^{2}}{K_{d r}}$ to $S$ in the reservoir module, leading to a modified compressibility term $S^{*}=S+\frac{\left(\alpha^{n-1}\right)^{2}}{K_{d r}}$. Consecutively, the reservoir equations are solved as follow:

Solving the reservoir equations: For a given $\delta \phi^{* m-1}$, the porosity $\phi^{n, m}$ is approximated as $\phi^{n, m} \approx \phi^{\circ}+\delta \phi^{* m-1}+S^{*}\left(p^{n, m}-p^{\circ}\right)$, allowing to compute implicitly $p^{n, m}$ because the approximated porosity depends only on pressure data. In other words the total mean stress and the plastic porosity do not change during solving the reservoir iterations, i.e. $\sigma_{t v}^{n, m} \approx \sigma_{t v}^{n-1}$ and $\phi_{p}^{n, m} \approx \phi_{p}^{n-1}$. It means that the term $\delta \phi_{m a t r i x}^{n, m}$ is explicitly approximated by an expression that only depends on pressure data, as follows:

$$
\delta \phi_{\text {matrix }}^{n, m} \approx \delta \phi^{* m-1}+\frac{\left(\alpha^{n-1, m-1}\right)^{2}}{K_{d r}}\left(p^{n, m}-p^{\circ}\right)
$$

Consecutively, the pressure is transferred to the geomechanic module and the equations are solved as follows:

Solving the geomechanic equations: Once the pressure $p^{n, m}$ is determined, the term $\delta \phi^{* m}$ is computed implicitly using the expression:

$$
\delta \phi^{* m}=\frac{\left(\alpha^{n-1}\right)^{2}}{K_{d r}}\left(\sigma_{t v}^{n, m}-\sigma_{t v}^{\circ}\right)+\phi_{p}^{n, m}-\phi_{p}^{\circ}
$$

The Fixed-stress split consists of approximating solutions for reservoir and geomechanic equations in a sequential manner. The information being transferred between both modules are $p^{n, m}$ and $\delta \phi^{* m}$ respectively. This strategy has the advantage of coupling two different simulators in a very simple manner. For the geomechanical problem, only the pressure field is inserted as a volume force. For the reservoir problem, it is applied $\delta \phi^{*}$ as a porosity correction and $S^{*}$ as modified compressibility term.

Remark 1: The expression that computes the Biot's coefficient as a function of the ratio of the elastoplastic and elastic bulk modulus of the grains (28) is the main difference between the approaches provided by [28] and [59], where $\alpha$ is computed as a function of the elastoplastic deformation as a separate constitutive law. Kim et al. [62] suggest the computation for the elastoplastic bulk modulus $K_{d r_{e p}}$ using the expression (27). It is opted to maintain the plastic porosity and Biot coefficient constant during a timestep iteration. Only between time steps $\phi_{p}$ and $\alpha$ are updated. In particular, the coefficient $\alpha$ is updated using the expression (28). A similar approach is used in the references Kim et al. [27, 28, 62].

The validity of this scheme is tested against the fully coupled poroelastoplastic solution when applied to a two-dimensional vertical wellbore problem. 


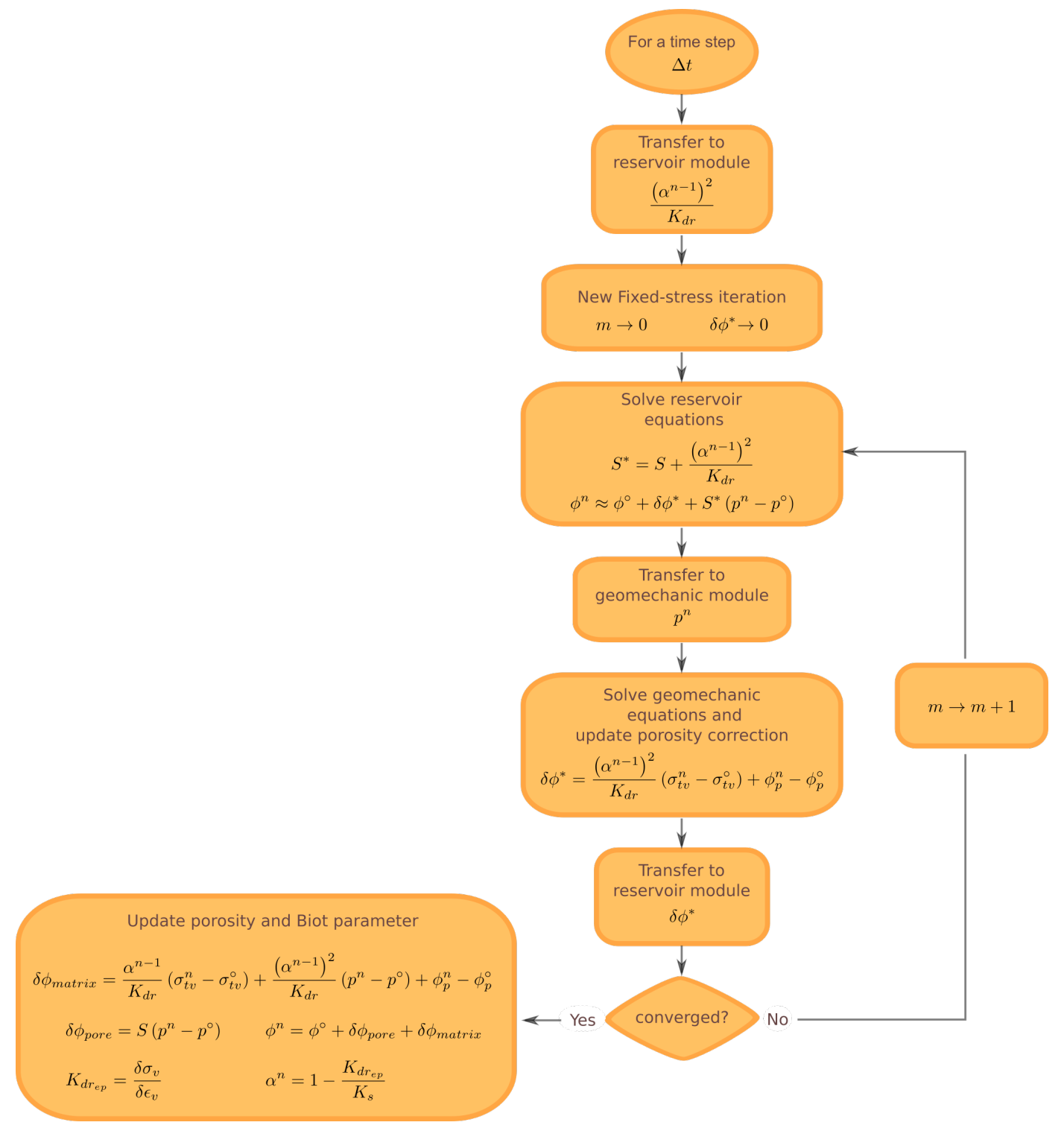

Figure 2: Elastoplastic fixed-stress split flowchart.

\subsection{Sequential fully implicit algorithm (SFI)}

The sequential fully implicit (SFI) algorithm is a common approach to handle coupled flow and transport equations for multiphase flow in porous media [30]. The SFI algorithm is applied with the fixed stress split as a robust procedure to approximate solutions of non-linear equations. It is considered, poroelastoplastic material behavior and the dependence of the permeability on the porosity. The algorithm 1 computes a new state $(\mathbf{u}, \mathbf{q}, p)^{m}$ in a time step of size $\Delta t$, by applying an external loop with counter $m$ and the maximum number of iterations $m_{f s s}$ to execute a sequence of two nonlinear solvers (a reservoir module and a geomechanics module). Information transfer between both solvers is performed using integration points transfer operations. The state of $\frac{\left(\alpha^{n-1}\right)^{2}}{K_{d r}}$ and the field $\delta \phi^{* m-1}$ is transferred to the reservoir module. Later, the first nonlinear solver computes the new state of the pair $(\mathbf{q}, p)^{m}$ considering $\delta \sigma_{t v}=0$. For the second solver, the pressure is transferred to the geomechanics module and $\delta \phi^{* m}$ is computed. The sequence is repeated until a desired stopping criteria is reached as a function of the variation of both sets of 
variables.

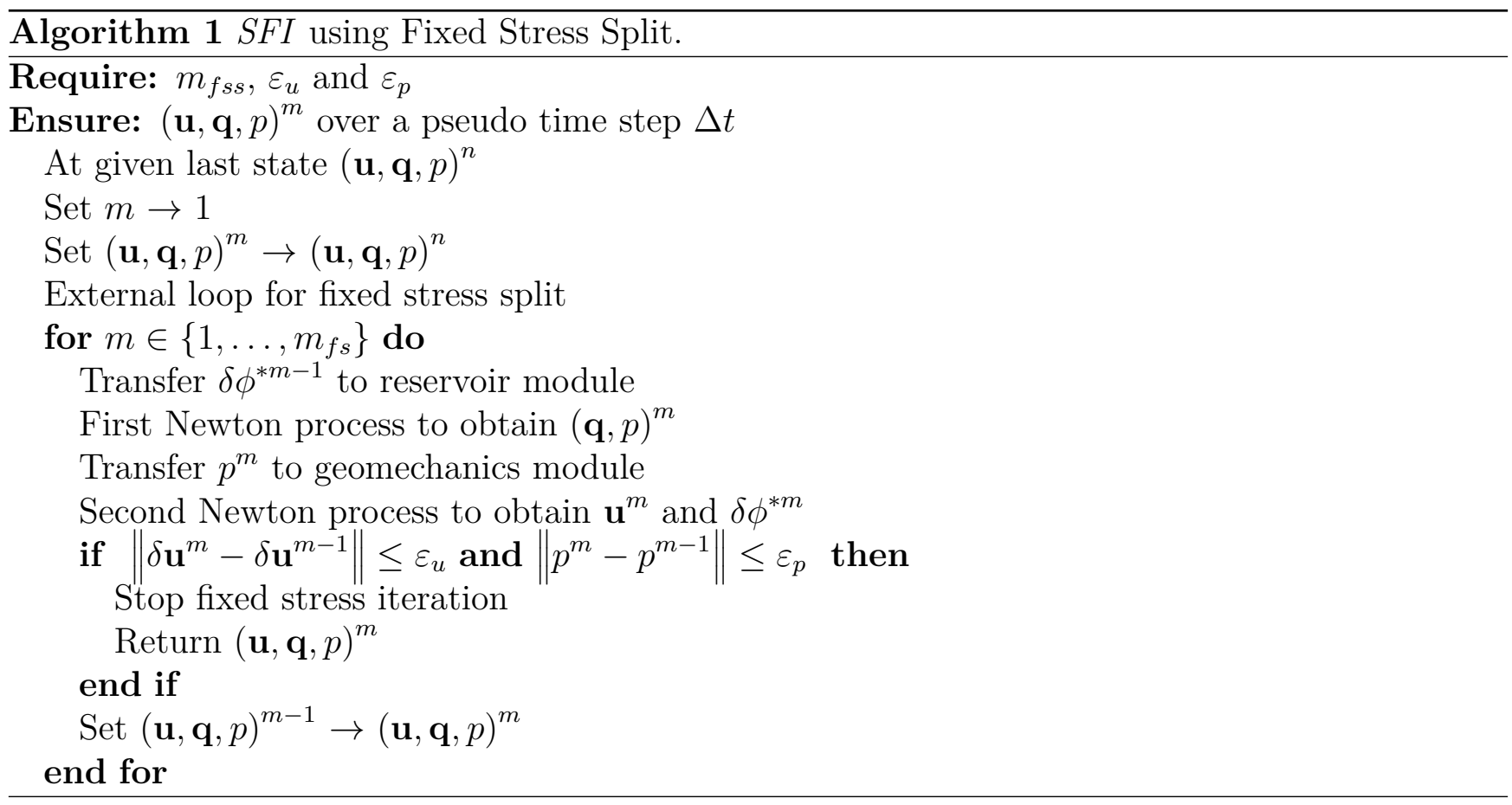

\subsection{Information transfer between modules}

The implementation of the SFI method offers modular programming and allows to select a proper numerical approach for generating approximations for both the reservoir and geomechanics modules. However, the SFI algorithm requires two information transfers between the modules at each iteration. There is a vast literature on sequential schemes, but in the best of our knowledge, there is a lack of explanation in how the modules are integrated. For completeness, it is provided some comments about a transfer interface being adopted.

The performance for the SFI procedure depends on the efficiency of each module independently an on the efficiency of the transfer of information. A simple shared memory management interface is applied to perform the transfer of information. The finite element discretizations adopted in subsections 2.4.1 and 2.4.2 store the information of the stress, deformation, pressure, and flux in computational memory objects associated with the integration point. Figure 3 provides a schematic representation for the transfer process in a finite element during the execution of the SFI procedure. The integration point transfer depends on the following rules:

- The geometric partition $\mathcal{T}_{H}$ is the same for both the reservoir and geomechanics problems;

- The numerical integration of the finite elements should have the same integration point indexation, between the reservoir and geomechanics modules;

- The reservoir and geomechanics modules can have arbitrary polynomial approximation order as long as they share the same order of numerical integration.

The steps for implementing the shared memory management interface are:

- For each finite element, the order of integration is the maximum required order of integration between the corresponding reservoir and geomechanics element; 
- The integration points are indexed;

- A memory object is associated with every integration point (see figure 3) where the necessary information for the geomechanical and fluid flow problem is stored;

- During the execution for the SFI, the geomechanics computation uses the information of the fluid and updates the information of the geomechanical computation (e.g. $\sigma_{v}, \nabla u, \phi_{p}$ ) (blue arrow in figure 3);

- The reservoir solution process uses the information of the geomechanics and updates the information of the fluid flow problem (e.g. p) (blue arrow in figure 3);

- The convergence of $S F I$ can be checked by taking the maximum change of state variables stored in the memory objects.

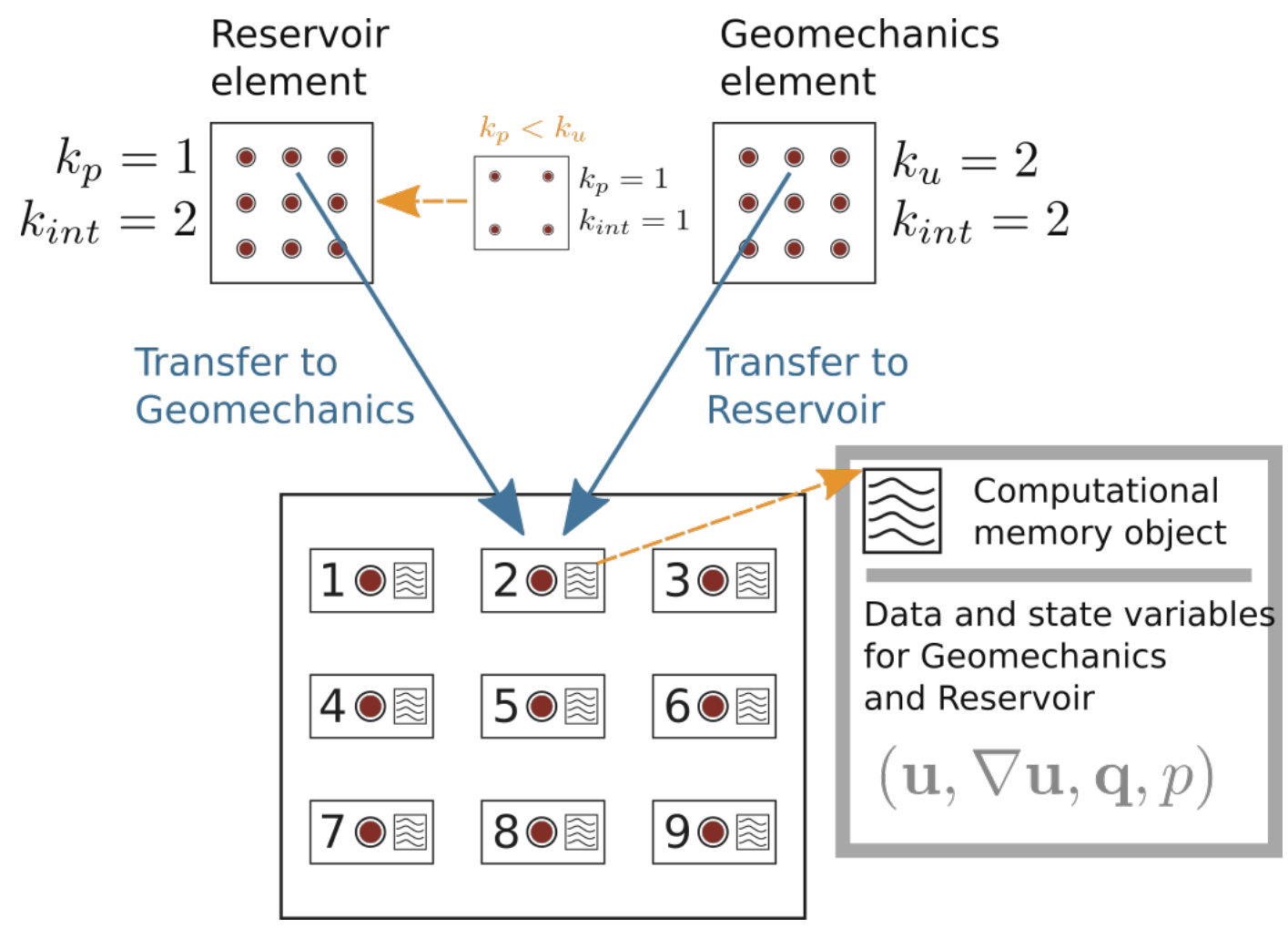

Figure 3: Schematic transfers operations during an SFI iteration.

\subsection{Nonlinear acceleration techniques}

The resolution for each problem considered in the SFI process uses Newton's method. A contribution of this research is the acceleration of the external loop of algorithm 1 in order to reduce the number of iterations associated with a time step. The Newton iterations are replaced by the modified Thomas method [67] that has better convergence properties.

The accelerated convergence for the internal loop

Consider a nonlinear system of equations $F(\mathbf{x}): D \subseteq \mathbb{R}^{n} \rightarrow \mathbb{R}^{n}$ that is a continuously differentiable multivariable function. $F(\mathbf{x})=0$ represents a nonlinear system with $n$ equations and $n$ unknowns. 
Denoting $\delta \mathbf{x}$ as a correction and $\mathbf{x}^{0}$ as initial state variable. A Newton's correction is given as follows:

$$
\begin{cases}\delta \mathbf{x}\left(\mathbf{x}^{k-1}\right) & =-\nabla F\left(\mathbf{x}^{k-1}\right)^{-1} F\left(\mathbf{x}^{k-1}\right) \\ \mathbf{x}^{k} & =\mathbf{x}^{k-1}+\delta \mathbf{x}\left(\mathbf{x}^{k-1}\right)\end{cases}
$$

Cordero et al. [68] introduced an accelerated convergence for the Newton method employing a two-step iterative method. In that iterative method, a first step is to perform a second-order Newton step to evaluate the iteration function of a subsequent state(s) $\psi\left(\mathbf{x}^{k}\right)$. The second step is to perform another Newton step that combines the information of a subsequent state(s) and the value of the iterative function $\psi\left(\mathrm{x}^{k}\right)$ leading to better convergence. Xiao and Yin [69] extend that method to construct a family of convergent high-order methods.

In this research, the ideas of [68, 69] and [67] are combined to define a form the function $\psi\left(\mathbf{x}^{k}\right)$ leading the classical Newton method $\mathrm{M}_{1}$, and the modified Thomas method $\mathrm{M}_{T}$. Details of them are provided concisely.

Method $\mathbf{M}_{1} \quad$ A new state $\mathbf{y}^{k}$ is provided by the iteration function:

$$
\mathbf{y}^{k}=\psi\left(\mathbf{x}^{k-1}\right)=\mathbf{x}^{k-1}+\delta \mathbf{x}\left(\mathbf{x}^{k-1}\right)
$$

The Newton's method is clearly:

$$
\mathbf{x}^{k}=\mathbf{y}^{k}
$$

This method requires one assembly, linear solve, and one function evaluation per update.

Method $\mathbf{M}_{T}$ This method defines the iteration function as follows:

$$
\mathbf{y}^{k}=\psi\left(\mathbf{x}^{k-1}\right)=\mathbf{x}^{k-1}+\omega^{k-1} \delta \mathbf{x}\left(\mathbf{x}^{k-1}\right)
$$

With the new update state defined as follows:

$$
\mathbf{x}^{k}=\mathbf{y}^{k}+\omega^{k-1} \delta \mathbf{x}\left(\mathbf{y}^{k}\right)
$$

Where the factor $\omega$ is the so-called acceleration factor [67], defined as :

$$
\omega^{k}=\omega^{k-1}+\frac{\delta \mathbf{x}\left(\mathbf{x}^{k-1}\right) \cdot \delta \mathbf{x}\left(\mathbf{y}^{k}\right)}{\delta \mathbf{x}\left(\mathbf{x}^{k-1}\right) \cdot \delta \mathbf{x}\left(\mathbf{x}^{k-1}\right)}
$$

The initial acceleration factor is $\omega^{0}$ is set to 1.0 at the beginning of the iterative process. This method provides a better convergence but requires two assemblies, two linear solve and two function evaluation per update, i.e. two Newton corrections. Different high order methods are provided by [69] with varying extra evaluations of the function and system inversions. Most higher- order methods have the inconvenience of requiring the computation of several updates. In 3D simulations, the inversion of a large Jacobian matrix can be restrictive.

Quasi-Newton methods result in lower convergence rates but have lower cost of execution. There are several options when Quasi-Newton approaches are adopted, as documented in [70, 71, 72]. It is employed a Quasi-Newton strategy forthcoming from the ideas provided by Sloan et al. 
[67]. The subproblems are solved using a single assembly for the global operator. In general, the correction is defined as:

$$
\begin{cases}\delta \mathbf{x}_{Q N}\left(\mathbf{x}^{k-1}\right) & =-\nabla F\left(\mathbf{x}^{s}\right)^{-1} F\left(\mathbf{x}^{k-1}\right) \\ \mathbf{x}^{k} & =\mathbf{x}^{k-1}+\delta \mathbf{x}_{Q N}\left(\mathbf{x}^{k-1}\right)\end{cases}
$$

The Quasi-Newton method denoted $\mathrm{QNM}_{T}$ is built using the structure of $\mathrm{M}_{T}$ considering 54 instead of 48 .

Where $\mathbf{x}^{s}$ is the unknown variable at some frozen state, for instance, the initial state of any time step. Details for such path are provided for each subproblem as follows:

Solving the reservoir equation $\left.r_{p}^{i}\right|^{n} \quad$ Considering the nonlinearities for the permeability introduced in previous sections the Quasi-Newton strategy adopted here for the reservoir equations is described as:

(i) Apply the Newton method during the first two iterations, one for capture the main variation for the pressure field, and the second one to capture the main permeability variations over a time step. Next, the last(inverted) Jacobian is frozen for the subsequent Quasi-Newton iterations;

(ii) Converge the solution as a series of Quasi-Newton updates using $\mathrm{QNM}_{1}$ or $\mathrm{QNM}_{T}$ technique.

Solving the geomechanics problem $\left.r_{u}^{i}\right|^{n} \quad$ Probably the most natural strategy for a QuasiNewton method for elastoplasticity is:

(i) Assemble the linear elastic global operator and decompose the Jacobian matrix;

(ii) Converge the solution as a series of Quasi-Newton updates without updating the Jacobian matrix and apply $\mathrm{QNM}_{1}$ or $\mathrm{QNM}_{T}$ acceleration. This approach is known as a secant method or initial stiffness scheme for elastoplasticity [67].

The acceleration properties for the scheme $\mathrm{QNM}_{T}$ will be documented in terms of number of iterations and normalized variables and compared to the unmodified scheme $\mathrm{QNM}_{1}$.

Nonlinear acceleration for external loop

The acceleration for the external loop is supported on the existence of a nonlinear function $\Psi(\mathbf{x})$, that provides a new value of $\mathbf{x}$. In the sense of a convergent fixed-point iteration a new updated variable $\mathbf{x}^{i}$ is obtained from a previous information $\mathbf{x}^{i-1}$ as follows:

$$
\mathrm{x}^{i}=\Psi\left(\mathrm{x}^{i-1}\right)
$$

Where $i=\{0,1, \ldots, n\}$. After $n$ iterations for the solution process it is obtained a sequence of states $s=\left\{\mathbf{x}^{0}, \mathbf{x}^{1} \ldots, \mathbf{x}^{n}\right\}$. For instance the function $\Psi\left(\mathbf{x}^{i-1}\right)$ can be represented by iterative processes like the Quasi-Newton methods introduced previously. Thus, the iterative process represented by 55 makes use of plenty of external iterations, especially for the Quasi-Newton case. There are several techniques for accelerate sequences provided by an arbitrary convergent process 55. that point to decrease the number of total iterations necessary to reach certain stop criterion.

Macleod [73] reviewed nine different algorithms for the acceleration of vector sequences, among which just Aitken's and Anderson's process were implemented. These algorithms are also identified as the First Difference Modulation $(F D M)$ and Second Difference Modulation SDM respectively. Their acceleration properties were analyzed by Jennings [38]. 
First Difference Modulation FDM Jennings [38], Macleod [73] define the transformation sequence as:

$$
\begin{aligned}
\mathbf{x}^{i} & =\Psi\left(\mathrm{x}^{i-1}\right) \\
\omega & =\frac{\left(\mathrm{x}^{i}-\mathrm{x}^{i-1}\right) \cdot\left(\mathrm{x}^{i-1}-\mathrm{x}^{i-2}\right)}{\left(\mathrm{x}^{i-1}-\mathrm{x}^{i-2}\right) \cdot\left(\mathrm{x}^{i}-2 \mathrm{x}^{i-1}+\mathrm{x}^{i-2}\right)} \\
\overline{\mathbf{x}}^{i} & =F D M\left(\mathrm{x}^{i-2}, \mathrm{x}^{i-1}, \mathrm{x}^{i}\right)=\mathrm{x}^{i}-\omega\left(\mathrm{x}^{i}-\mathrm{x}^{i-1}\right) \\
\mathbf{x}^{i} & =\overline{\mathbf{x}}^{i}
\end{aligned}
$$

Second Difference Modulation $\boldsymbol{S D M}$ Anderson [74], Jennings [38], Macleod [73] present the following alternative:

$$
\begin{aligned}
\mathbf{x}^{i} & =\Psi\left(\mathrm{x}^{i-1}\right) \\
\omega & =\frac{\delta \mathbf{x}^{i} \cdot\left(\delta \mathbf{x}^{i}-\delta \mathbf{x}^{i-1}\right)}{\left\|\delta \mathbf{x}^{i}-\delta \mathbf{x}^{i-1}\right\|^{2}} \\
\overline{\mathbf{x}}^{i} & =S D M\left(\mathbf{x}^{i-2}, \mathbf{x}^{i-1}, \mathbf{x}^{i}\right)=\mathbf{x}^{i}+\omega\left(\mathbf{x}^{i-1}-\mathbf{x}^{i}\right) \\
\mathbf{x}^{i} & =\overline{\mathbf{x}}^{i}
\end{aligned}
$$

Where $\delta \mathbf{x}^{i}=\mathbf{x}^{i}-\mathbf{x}^{i-1}$.

Given a squence $\left\{\mathbf{x}^{i-2}, \mathbf{x}^{i-1}, \mathbf{x}^{i}\right\}$, scalar $\omega$, and two transformations $\mathbf{u}$ and $\mathbf{v}$ :

$$
\begin{gathered}
\mathbf{u}=\mathbf{x}^{i-1}+\omega\left(\mathbf{x}^{i-2}-\mathbf{x}^{i-1}\right) \\
\mathbf{v}=\mathbf{x}^{i}+\omega\left(\mathbf{x}^{i-1}-\mathbf{x}^{i}\right)
\end{gathered}
$$

The corresponding $\omega$ value is chosen to minimize $\|\mathbf{u}-\mathbf{v}\|^{2}$.

It is important to point out that $F D M$ and $S D M$ require three previous states.

A remarkable transformation of this kind is the so-called Shanks transformation ([40, 75]) that is an all-purpose method for the acceleration of convergent sequences.

The modified iterative process considering indistinctly FDM, SDM or another kind of sequence transformation/acceleration of the external loop can be presented in the following script:

1. Get initial guest $\mathbf{x}^{0}$ and set $i=1$;

2. Compute $\mathbf{x}^{i}=\Psi\left(\mathbf{x}^{i-1}\right)$;

3. If $i \geq n_{\text {accel }}$, then apply the transformation with $n_{\text {accel }}$ states available to obtain the modified state $\overline{\mathbf{x}}^{i}$

4. Set $\mathbf{x}^{i}=\overline{\mathbf{x}}^{i}$

5. Set $i \rightarrow i+1$;

6. Repeat step 2 to 5 till convergence is reached. 
Shanks augmented acceleration for $\boldsymbol{F} \boldsymbol{D} \boldsymbol{M} / \boldsymbol{S D} \boldsymbol{M}$ The mathematician Daniel Shanks have been defined a recursive technique for improving the acceleration of divergent or slowly convergent sequences. The proposed augmented acceleration is inspired by the example provided in [40], when a slowly convergent series to approximate number $\pi$ is accelerated by means of a recursive procedure. During the execution for the nonlinear process $\Psi\left(\mathrm{x}^{i-1}\right)$ a series of states $\left\{\mathbf{x}^{1}, \mathbf{x}^{2} \ldots, \mathbf{x}^{i}, \ldots \mathbf{x}^{n}\right\}$ are generated as the operations occur. The transformations FDM and SDM are applied recursively as more states are available to construct a better modified state $\overline{\mathbf{x}}^{i}$. For brevity let denote the transformation $T$ that requires three states and provided a new modified state $\overline{\mathbf{x}}_{i, i-1, i-2}$ as $\overline{\mathbf{x}}_{i, i-1, i-2}=\mathrm{T}\left(\mathbf{x}^{i-2}, \mathbf{x}^{i-1}, \mathbf{x}^{i}\right)$. A new two Shanks transformations $\overline{\mathbf{x}}^{2}$ and $\overline{\mathbf{x}}^{3}$ that account for extra available states is introduced as:

$$
\overline{\mathbf{x}}_{i, i-1, i-2}^{2}=\mathrm{T}\left(\overline{\mathbf{x}}_{i, i-1, i-2}, \overline{\mathbf{x}}_{i-1, i-2, i-3}, \overline{\mathbf{x}}_{i-2, i-3, i-4}\right)
$$

and

$$
\overline{\mathbf{x}}_{i, i-1, i-2}^{3}=\mathrm{T}\left(\overline{\mathbf{x}}_{i, i-1, i-2}^{2}, \overline{\mathbf{x}}_{i-1, i-2, i-3}^{2}, \overline{\mathbf{x}}_{i-2, i-3, i-4}^{2}\right)
$$

\begin{tabular}{|c|c|c|c|c|}
\hline$i$ & $\Psi\left(\mathbf{x}^{i}\right)$ & $\overline{\mathbf{x}}$ & $\overline{\mathbf{x}}^{2}$ & $\overline{\mathbf{x}}^{3}$ \\
\hline \hline 1 & $\mathbf{x}^{1}=\Psi\left(\mathbf{x}^{0}\right)$ & $\mathrm{N} / \mathrm{A}$ & $\mathrm{N} / \mathrm{A}$ & N/A \\
\hline 2 & $\mathbf{x}^{2}=\Psi\left(\mathbf{x}^{1}\right)$ & N/A & N/A & N/A \\
\hline 3 & $\mathbf{x}^{3}=\Psi\left(\mathbf{x}^{2}\right)$ & $\overline{\mathbf{x}}_{3,2,1}$ & N/A & N/A \\
\hline 4 & $\mathbf{x}^{4}=\Psi\left(\overline{\mathbf{x}}_{3,2,1}\right)$ & $\overline{\mathbf{x}}_{4,3,2}$ & N/A & N/A \\
\hline 5 & $\mathbf{x}^{5}=\Psi\left(\overline{\mathbf{x}}_{4,3,2}\right)$ & $\overline{\mathbf{x}}_{5,4,3}$ & $\overline{\mathbf{x}}_{5,4,3}^{2}$ & N/A \\
\hline 6 & $\mathbf{x}^{6}=\Psi\left(\overline{\mathbf{x}}_{5,4,3}^{2}\right)$ & $\overline{\mathbf{x}}_{6,5,4}$ & $\overline{\mathbf{x}}_{6,5,4}^{2}$ & N/A \\
\hline 7 & $\mathbf{x}^{7}=\Psi\left(\overline{\mathbf{x}}_{6,5,4}^{2}\right)$ & $\overline{\mathbf{x}}_{7,6,5}$ & $\overline{\mathbf{x}}_{7,6,5}^{2}$ & $\overline{\mathbf{x}}_{7,6,5}^{3}$ \\
\hline 8 & $\mathbf{x}^{8}=\Psi\left(\overline{\mathbf{x}}_{7,6,5}^{3}\right)$ & $\overline{\mathbf{x}}_{8,7,6}$ & $\overline{\mathbf{x}}_{8,7,6}^{2}$ & $\overline{\mathbf{x}}_{8,7,6}^{3}$ \\
\hline$\vdots$ & $\vdots$ & $\vdots$ & $\vdots$ & $\vdots$ \\
\hline$i$ & $\mathbf{x}^{i}=\Psi\left(\overline{\mathbf{x}}_{i, i-1, i-2}^{3}\right)$ & $\overline{\mathbf{x}}_{i, i-1, i-2}$ & $\overline{\mathbf{x}}_{i, i-1, i-2}^{2}$ & $\overline{\mathbf{x}}_{i, i-1, i-2}^{3}$ \\
\hline
\end{tabular}

Table 3: Proposed Shanks-like transformations $\overline{\mathbf{x}}^{2}$ and $\overline{\mathbf{x}}^{3}$ sequences.

The table shows that Shanks transformations $\overline{\mathbf{x}}^{2}$ and $\overline{\mathbf{x}}^{3}$ require, 5 and 7 additional states. These transformations enhance the acceleration of $\mathrm{T}=\{F D M, S D M\}$. The improved convergence is shown later through several numerical examples.

\subsection{Enhanced SFI algorithm}

Few methods were documented to improve the performance of the external loop for obtaining poroelastoplastic approximated solutions. Jiang and Tchelepi [30] presented several acceleration techniques applied to the $S F I$ in the context of the multiphase flow in porous media where they applied Anderson Acceleration, Quasi-Newton and Aitken's technique. Jiang and Tchelepi [30] simulated several 2D reservoir cases and demonstrated that Aitken's technique results in better performance and convergence. In Both et al. [63] a robust linearization technique was incorporated in the Fixed stress split method using the Richards equation coupled with the linear elastic equations. It is an original contribution of this research to implement and compare two acceleration 
techniques to enhance the performance for the SFI with fixed stress iterations in the context of poroelastoplasticity using nonlinear acceleration techniques presented by Jennings [38]. The outer loop of the $S F I$ algorithm is accelerated with Shanks transformations.

The enhanced ESFI process use the formulae (57), (59), and their Shanks-like versions (62), and (63), leading to a substantial reduction of the number of iterations. The implementation of the enhanced sequential fully implicit $(E S F I)$ represents an improvement of the SFI by adding additional statements as is presented in the algorithm 2 .

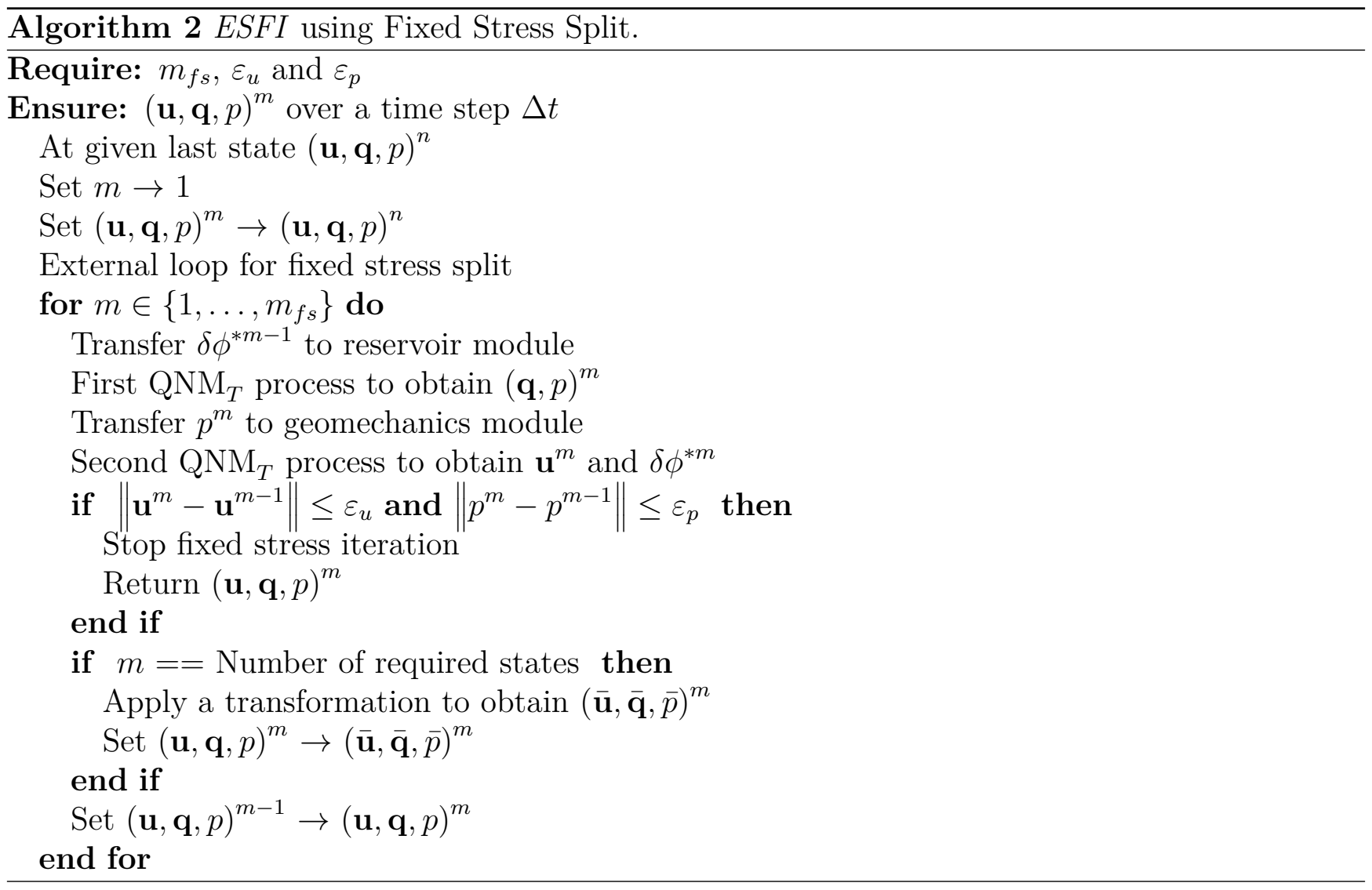

\section{Numerical results}

In this section, several numerical examples with different physical and constitutive modeling are provided. The objective is to demonstrate the correctness, robustness, and efficiency for the ESFI implementation. Two cases with analytic solutions under the assumption of linear constitutive modeling are considered; a one-dimensional Terzaghi's consolidation problem and the two-dimensional vertical wellbore problem are simulated to display the fidelity of the approximations. For the case of nonlinear constitutive modeling, an elastoplastic Mohr-Coulomb model is implemented to model the nonlinear rock deformation, and for the reservoir, a nonlinear permeability Petunin model is contemplated. The physical situation is a vertical producer wellbore under plane strain assumption. To corroborate the correctness in this nonlinear case, a Runge-Kutta approximation is performed on a set of equations written into the frame of cylindrical coordinates system and under axisymmetric conditions. In addition, a three-dimensional problem is presented to show the capability of the FEM implementation in dealing with geometrical 3D representations.

For both, the SFI and ESFI methods, the results are presented in terms of the number of iterations per time step of the inner loops (nonlinear problems) and number of iterations of the 
outer loop. Furthermore, a brief error analysis is provided for the Terzaghi's consolidation problem and the vertical wellbore to show the convergence properties of the selected schemes for time and space discretizations, respectively.

\subsection{One-dimensional Terzaghi's consolidation}

Terzaghi's classical consolidation problem is a simple one-dimensional problem with the known analytic solution. A concise description of the one-dimensional consolidation problem is provided in [49]. The physical process consider two stages: the first one is related the vertical loading to of a fully saturated soil/rock with impervious boundaries over pressurizing the fluid that dwell inside (Undrained response); the second stage represents the expulsion of the fluid through a boundary that suddenly became permeable leading to consolidation of the column, because the loading is progressively transferred to the matrix (Drained response). In the context of drilling operations a similar situation occurs when a drilling tool abruptly release an over pressurized cross-bedded layer or formation.

The boundary conditions for the consolidation problem are given in terms of pore pressure change $p$ respect to a hydrostatic condition and total stress. The vertical total stress $\sigma_{n}=\mathbf{t} \cdot \mathbf{n}$ (Load BC) and pressure change (Seepage BC) are prescribed over the top of the column. The other part of the boundary is zero normal displacements and zero normal flux (Impervious BC) as is shown in the figure 4. For the initial stage, the undrained response of the column is given by:

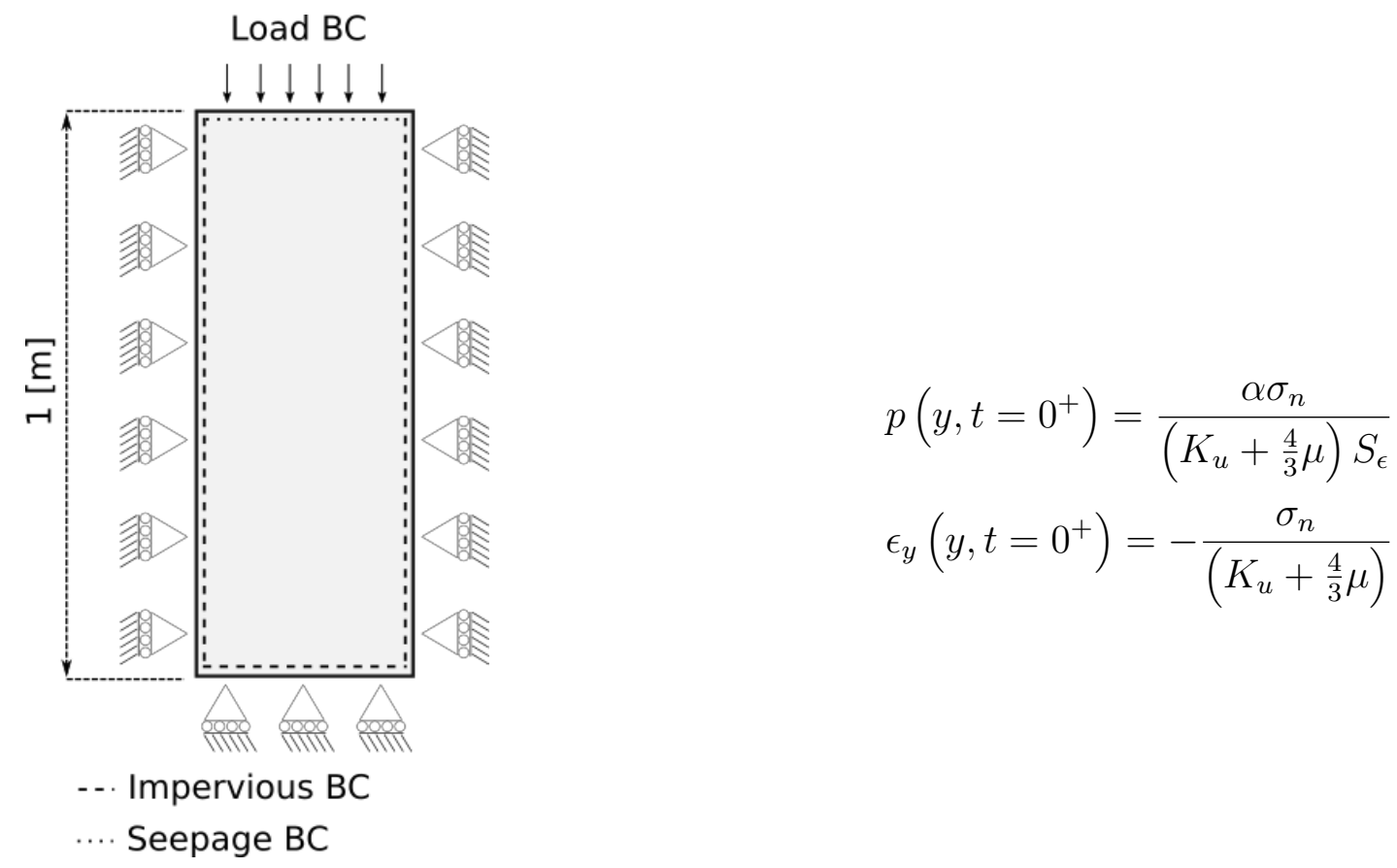

Figure 4: Domain and boundary conditions for one-dimensional consolidation.

Where the $K_{u}=K+\frac{\alpha^{2}}{S_{\epsilon}}$ represents the undrained bulk modulus. For the second stage, the solution for a finite column of height $h$ is expressed in terms of infinite series:

For the dimensionless pore pressure change:

$$
\bar{p}(\bar{y}, \bar{t})=\sum_{n=0}^{n=\infty}\left(\frac{4}{\pi(2 n+1)}\right) \sin \left(\frac{(2 n+1) \pi}{2} \bar{y}\right) \exp \left(-\frac{(2 n+1)^{2} \pi^{2}}{4} \bar{t}\right)
$$


For the dimensionless vertical displacement:

$$
\bar{u}_{y}(\bar{y}, \bar{t})=1-\bar{y}-\sum_{n=0}^{n=\infty}\left(\frac{8}{\pi^{2}(2 n+1)^{2}}\right) \cos \left(\frac{(2 n+1) \pi}{2} \bar{y}\right) \exp \left(-\frac{(2 n+1)^{2} \pi^{2}}{4} \bar{t}\right)
$$

where the dimensionless groups are composed as follow:

$$
p=\frac{\alpha \sigma_{n}}{\left(K_{u}+\frac{4}{3} \mu\right) S_{\epsilon}} \bar{p} \quad y=h \bar{y} \quad t=\frac{h^{2}}{c_{h}} \bar{t} u_{y}=h \bar{u}_{y}
$$

Remembering that the diffusion coefficient $c_{h}\left[\mathrm{~m}^{2} \mathrm{~s}^{-1}\right][49]$ :

$$
c_{h}=\frac{\kappa}{\eta S_{\epsilon}} \frac{K+\frac{4}{3} \mu}{K_{u}+\frac{4}{3} \mu}
$$

\subsubsection{Time discretization convergence rates}

Above all, the selected scheme for the time discretization is the Euler method. To show the correctness for the Euler scheme in time for both, the fully coupled solver $(F C)$ and $S F I$ solver, it was selected a high-order approximation in space, i.e. 20 quadrilateral finite elements with cubic and quadratic polynomial order for displacements and pressure, respectively. The material parameters are presented in table 4 . The linear convergence rate for the Euler scheme is obtained by setting $\Delta t$ to be $\{0.1,0.05,0.025,0.0125,0.00625\}[\mathrm{s}]$ (see figure 5 ). The final time $t_{f}=1.0[\mathrm{~s}]$ is used for all the simulations.

\begin{tabular}{|c|c|c|}
\hline Parameter & Variable [unit] & Value \\
\hline \hline Young's modulus & $E \mathrm{MPa}$ & 1000.0 \\
\hline Poisson's ratio & $\nu$ & 0.2 \\
\hline Biot's coefficient & $\alpha$ & 1 \\
\hline Fluid compressibility & $c_{f} \mathrm{MPa}^{-1}$ & 0 \\
\hline Fluid dynamic viscosity & $\eta \mathrm{Pa} \mathrm{s}^{\mathrm{s}}$ & $1 \times 10^{-3}$ \\
\hline Initial porosity & $\phi^{\circ}$ & 0.1 \\
\hline Initial Abs. permeability & $\kappa^{\circ} \mathrm{m}^{2}$ & $1 \times 10^{-13}$ \\
\hline Vertical total stress & $\sigma_{n} \mathrm{MPa}$ & 1.0 \\
\hline Initial pressure excess & $p^{\circ} \mathrm{MPa}$ & 1.0 \\
\hline Tolerance for stop criterion & $\varepsilon_{u}=\varepsilon_{p}$ & $1 \times 10^{-6}$ \\
\hline
\end{tabular}

Table 4: Parameters employed for Terzaghi consolidation test. 

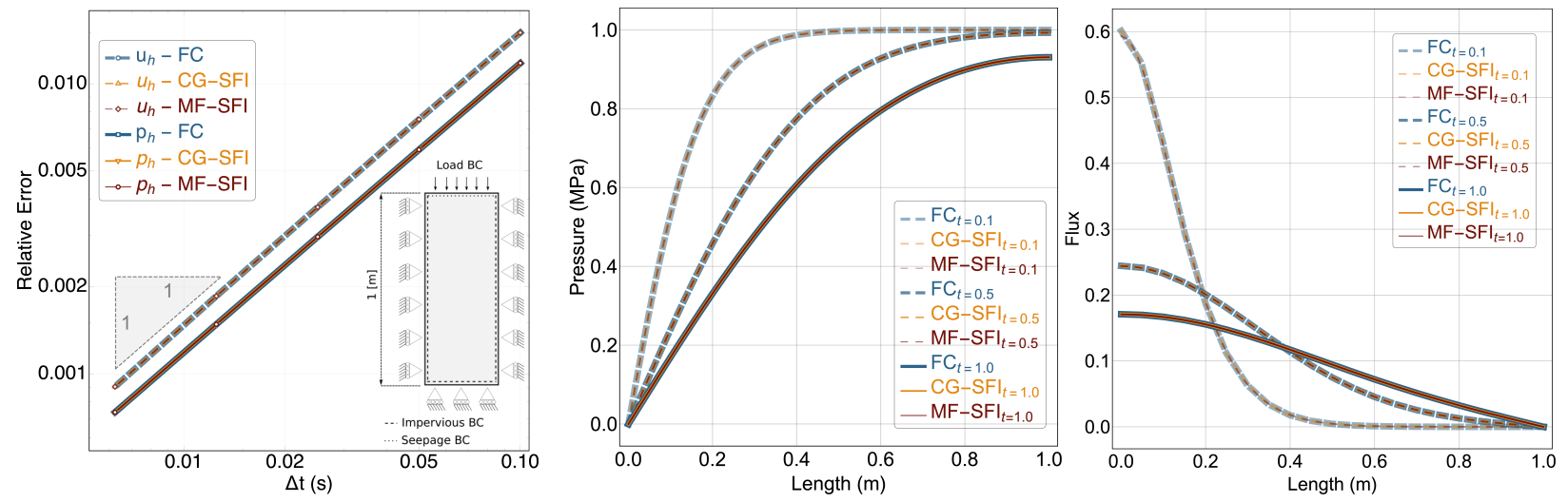

Figure 5: History of convergence in time, pressure and flux plots.

Figure 5 displays several solver configurations labeled as $F C$ fully coupled, $S F I$ with discrete weak statement I 2.4.1 CG-SFI, and SFI with discrete weak statement II 2.4.2 MF-SFI. The figure 5 shows a good match between the different operators and verify the correctness for the implementation of the SFI procedure in a linear setting.

One-dimensional comparison in a linear setting

Let define the performance ratio $r_{p}$ as:

$$
r_{p}=\frac{t_{E S F I}}{t_{S F I}}
$$

where $t_{E S F I}$ and $t_{S F I}$ are respectively, the computational CPU time during the performance of the ESFI and SFI methods. Thus, to compare the SFI and ESFI procedures it was considered the following directives:

- The simulation time corresponds to the execution of both SFI and ESFI. The reported average time value is computed as the mean of ten simulations;

- The quantity $r_{p}$ is computed as a ratio of the average time for each ESFI execution over the average time for each $S F I$;

- The reported iterations are associated with the external loop, and not for the internal loops because they are linear and they converge with a single Newton iteration;

- The external loop reaches the convergence when the relative variation of each state variable is less than the tolerance $\varepsilon$ (see algorithm 2). The tolerance for geomechanics and reservoir modules are $\varepsilon_{u}$ and $\varepsilon_{p}$, respectively. 

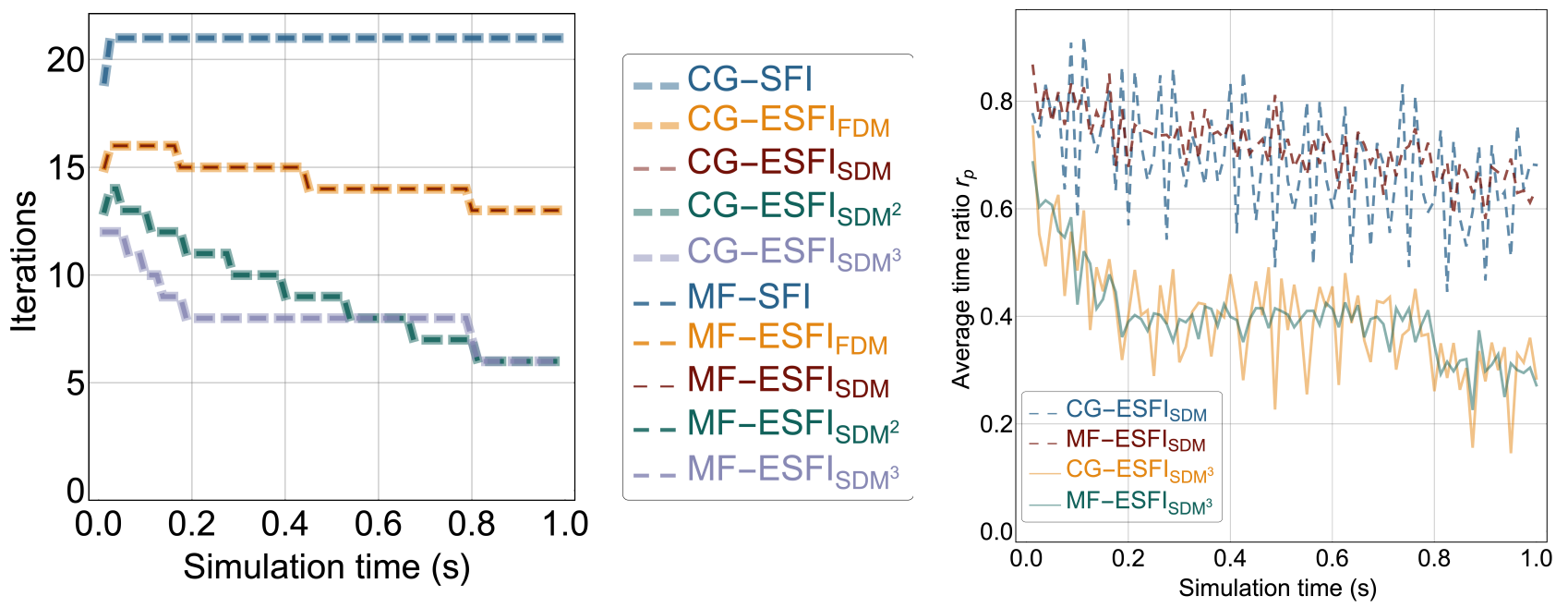

Figure 6: Number of iterations and performance ratio $r_{p}$ against simulation time.

The time step is regular and with $\Delta t=0.0125[\mathrm{~s}]$. Figure6 shows that for an linear operator the number of iterations used by the conventional $S F I$ procedure ( $C G$-SFI and $M F-S F I)$ is decreased. The usage of $F D M$, and $S D M$ for the linear case accelerate the solution process ending with the same number of iterations for any case. Their corresponding Shanks transformations $F D M^{2}, F D M^{3}$, $S D M^{2}$ and $S D M^{3}$ end into a reduced number of iterations. Figure 6 does not show the curves for $F D M^{2}$, and $F D M^{3}$ because they demand same number of iterations of $S D M^{2}$ and $S D M^{3}$. Respect to the $r_{p}$, the figure 6 shows that due to the reduction of external iterations the execution for ESFI is faster than $S F I$ leading to $r_{p}<1$.

\subsection{Drilling of a borehole}

Drilling is an inherent part of the exploration and reservoir production process. A simplified approach to model the drilling of a vertical borehole is to consider an instantaneous material removal, the weight of the overburden rocks and the stress state of the side burden rocks are accounted through an initial stress field, that some times are approximated as a hydrostatic stress field. Two physical situations that discern on being linear or not are presented.

\subsubsection{Convergence rates for spatial discretization}

For show the spatial approximation rates of the selected finite element scheme, two uncoupled problems with the data in Table 5 are presented in terms of the corresponding function space configuration:

For the geomechanics problem, it is selected the following analytic displacement expression:

$$
\mathbf{u}(r)=\frac{\sigma_{w b}+\sigma^{\circ}}{2 \mu} \frac{r_{w}^{2}}{r}
$$

For the reservoir problem, it is chosen the following analytic pressure expression:

$$
p(r)=p_{w b}+\frac{p^{\circ}-p_{w b}}{\ln \left(\frac{r_{e}}{r_{w}}\right)} \ln \left(\frac{r}{r_{w}}\right)
$$

Let $\mathcal{L}$ and $\mathrm{Q}$ stand for linear, and quadratic approximation orders, and let the subscripts $\mathrm{H}^{1}$ and $\mathrm{H}$ (div) stand for Continuous Galerkin and for mixed finite element approximation. In figures 
7 and $8 \mathcal{Q}_{\mathrm{H}^{1}} \mathcal{L}_{\mathrm{H}^{1}}$ represents the approximation space configuration with quadratic $\mathrm{H}^{1}$ elements for displacements and linear $\mathrm{H}^{1}$ elements for pore pressure; $\mathcal{Q}_{\mathrm{H}^{1}} \mathcal{L}_{\mathrm{H}(\mathrm{div})}$ stands for quadratic $\mathrm{H}^{1}$ elements for displacements and linear mixed finite elements for fluid velocity and pore pressure.
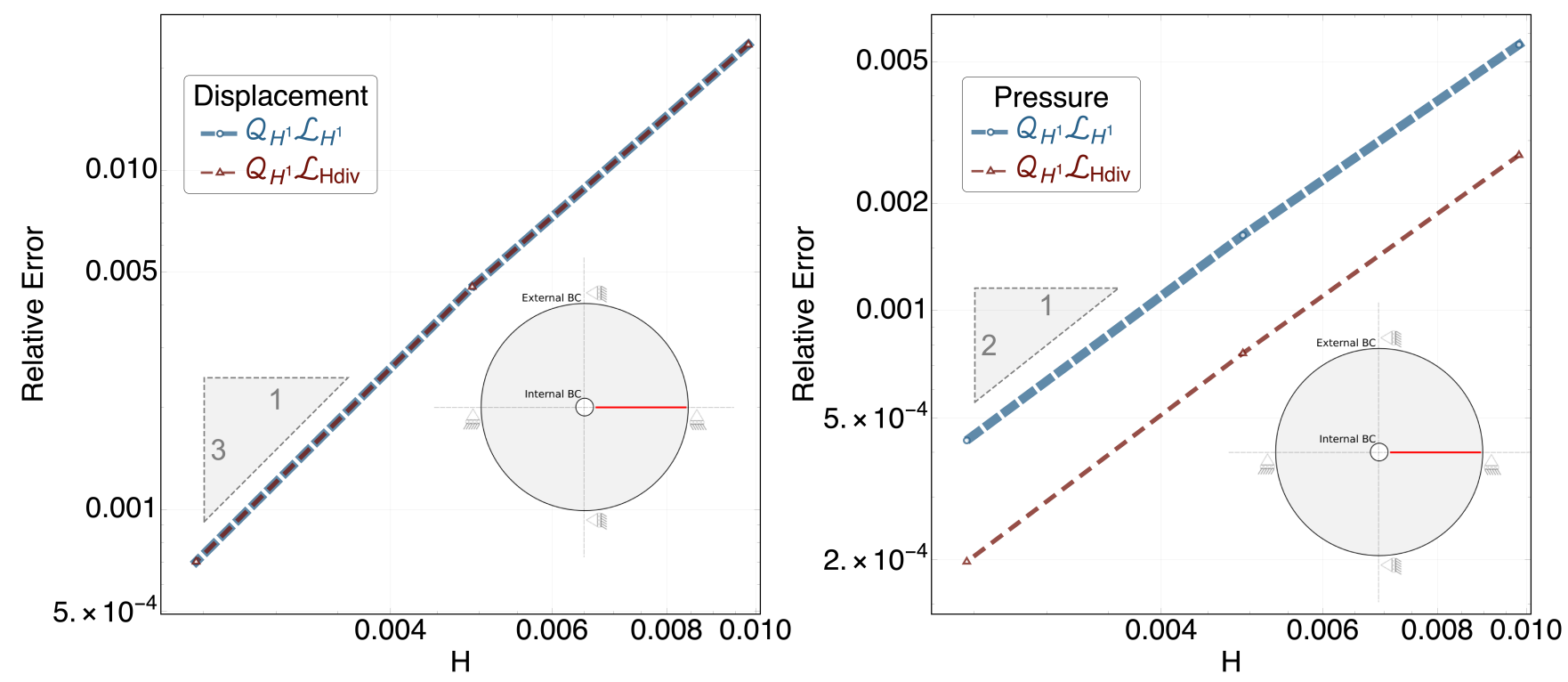

Figure 7: History of convergence in space, displacement and pressure.

Figure 7 shows that the approximation rates for $\mathbf{u}$ and $p$ are $k+1$, where $k$ represents polynomial order for the state variable. In particular the displacement approximation is the same for both, $\mathcal{Q}_{\mathrm{H}^{1}} \mathcal{L}_{H^{1}}$ and $\mathcal{Q}_{\mathrm{H}^{1}} \mathcal{L}_{\mathrm{H}(\mathrm{div})}$ because the approximation order is equal, in other words the lines of Figure 7 on the right are parallel in the asymptotic case $\mathrm{H} \rightarrow 0$.

Figure 8 shows the approximation rate and a plot over line for the flux variable q. For the case $\mathcal{Q}_{\mathrm{H}^{1}} \mathcal{L}_{\mathrm{H}^{1}}$ the approximation order is $k$, but with $\mathcal{Q}_{\mathrm{H}^{1}} \mathcal{L}_{\mathrm{H}(\text { div) }}$ the approximation order is $k+1$ (see Figure 8 on the left.). It is important to point out that for $\mathcal{Q}_{\mathrm{H}^{1}} \mathcal{L}_{\mathrm{H}^{1}}$ the approximation is linear and then the flux is constant piecewise, but for $\mathcal{Q}_{\mathrm{H}^{1}} \mathcal{L}_{\mathrm{H}(\mathrm{div})}$ the flux approximation is local conservative and clearly more accurate. Figure 8 on the right shows graphically this characteristic. As can be expected based on their accurate flux approximation and pointing for future developments in the sense of multiphase flow, a particular attention is invested for results with mixed formulation more than continuous formulation for Darcy flow.

In addition Figures 7 and 8 report the characteristic of the finite element approximations with two different polynomials orders that are attached to the LBB inf-sup condition, i.e. the polynomial order for displacement should be one order higher than the pressure approximation. Under these circumstances and results it was confirmed the correctness of the implementation for the spatial approximation scheme presented in sections 2.4.1 and 2.4.2. 

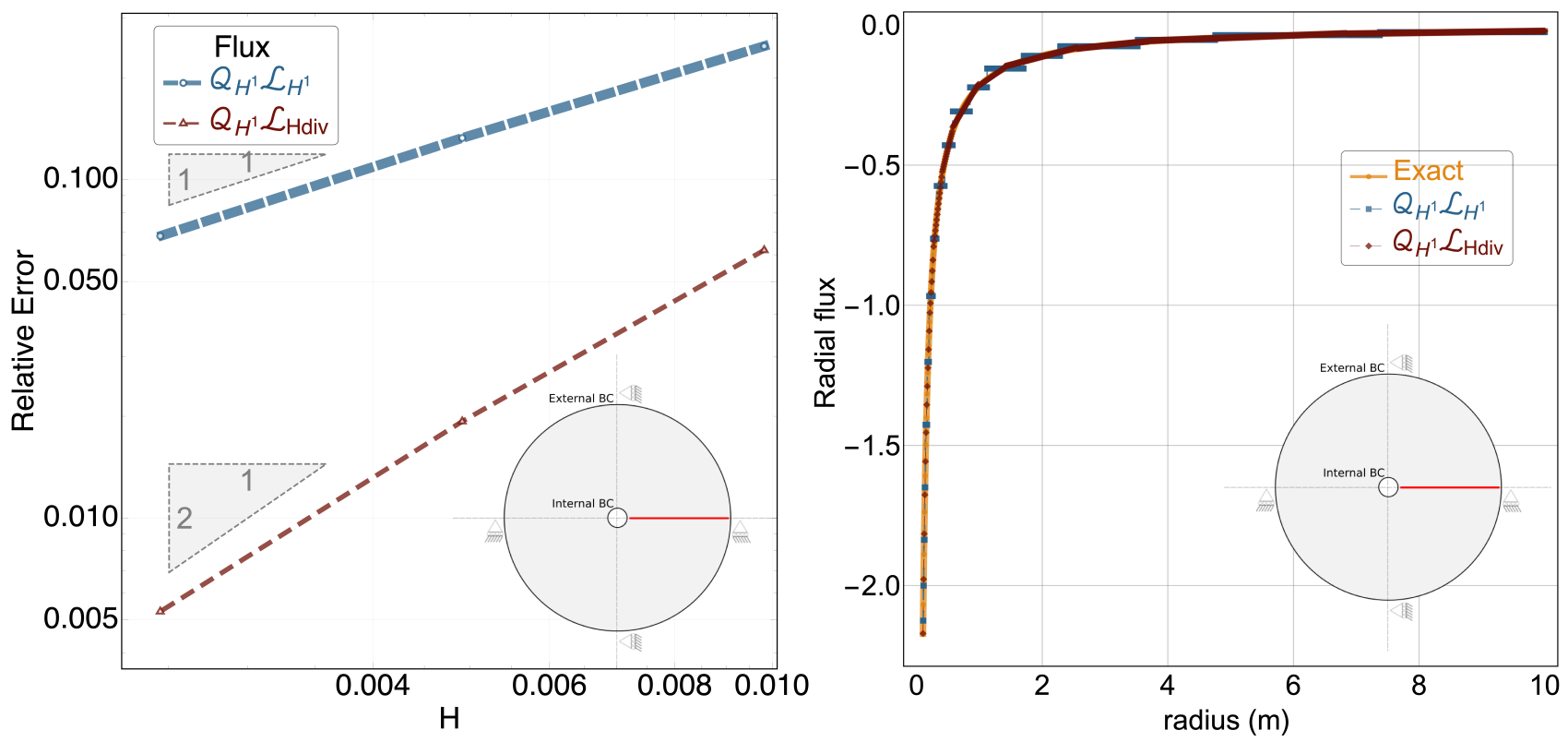

Figure 8: History of convergence in space, flux and radial flux plot over red line.

\begin{tabular}{|c|c|c|}
\hline Parameter & Variable [unit] & Value \\
\hline \hline Young's modulus & $E \mathrm{MPa}$ & 1000.0 \\
\hline Poisson's ratio & $\nu$ & 0.2 \\
\hline Biot's coefficient & $\alpha$ & 0 \\
\hline Fluid compressibility & $c_{f} \mathrm{MPa}^{-1}$ & 0 \\
\hline Fluid dynamic viscosity & $\eta \mathrm{Pa} \mathrm{s}^{\circ}$ & $1 \times 10^{-3}$ \\
\hline Initial porosity & $\phi^{\circ}$ & 0.1 \\
\hline Initial Abs. permeability & $\kappa^{\circ} \mathrm{m}^{2}$ & $1 \times 10^{-13}$ \\
\hline Initial hydrostatic total stress & $\sigma^{\circ} \mathrm{MPa}$ & -50 \\
\hline Initial pressure excess & $p^{\circ} \mathrm{MPa}$ & 30 \\
\hline Mud pressure & $p_{w b} \mathrm{MPa}$ & 20 \\
\hline Internal BC normal stress & $\sigma_{w b}$ & -20 \\
\hline Tolerance for stop criterion & $\varepsilon_{u}=\varepsilon_{p}$ & $1 \times 10^{-7}$ \\
\hline
\end{tabular}

Table 5: Parameters employed for computing approximation spatial rates.

\subsubsection{Transient linear case}

Considering a circular geometry and symmetry in cylindrical coordinates $\mathbf{x}(r, \theta, z)$, it can be obtained an analytic solution that only depend on the radius $r$. In this subsection is briefly described the analytical solution presented in [49].

The initial pore pressure is constant $p^{\circ}$ and the initial stress state is hydrostatic as follows:

$$
\boldsymbol{\sigma}(r, t=0)=\sigma^{\circ} \mathbf{I}
$$

After the drilling and with a mud cake form on the borehole newly created walls, the drilling fluid pressure inside the wellbore $p_{w b}$ is applied on wellbore walls.

For the initial time $t=0$, there is no variation of reservoir fluid pressure, nor volume change. It implies that the instantaneous response due to the application of $p_{w b}$ is undrained and then corresponds to a standard elastic solution: 


$$
\mathbf{u}\left(r, t=0^{+}\right)=\frac{p_{w b}+\sigma^{\circ}}{2 \mu} \frac{r_{w}^{2}}{r}
$$

Thus, the analytic expression represents the evolution of pore pressure and displacement fields beyond the undrained response till the corresponding drained limit. The general expressions for pore pressure and displacements are:

$$
\begin{gathered}
p(r, t)=p^{\circ}+\left(p_{w b}-p^{\circ}\right) \bar{p}(r, t) \\
\mathbf{u}(r, t)=\frac{p_{w b}+\sigma^{\circ}}{2 \mu} \frac{r_{w}^{2}}{r}+r_{w} \frac{\alpha\left(p_{w b}-p^{\circ}\right)}{K+\frac{4}{3} \mu} \overline{\mathbf{u}}(r, t)
\end{gathered}
$$

The full formulas for $\bar{p}(r, t)$ and $\overline{\mathbf{u}}(r, t)$ are based on integral expression of Bessel functions of the first and second kind. These integral expressions are provided in [49].

The early time solution $t \ll \frac{r_{w}^{2}}{c_{h}}$, i.e. $c_{h} \frac{t}{r_{w}^{2}} \rightarrow 0$ allows a simplification of the expressions $\bar{p}(r, t)$ and $\overline{\mathbf{u}}(r, t)$, by invoking the asymptotic expansions of Bessel functions, it can be obtained two simplified formulas:

$$
\begin{gathered}
\bar{p}(r, t)=\sqrt{\frac{r_{w}}{r}}\left\{\operatorname{erfc}(\tau)-\frac{r-r_{w}}{16 r}\left(\frac{2}{\sqrt{\pi}} \frac{\sqrt{c_{h} t}}{r_{w}} \exp \left(-\tau^{2}\right)-\frac{r-r_{w}}{r_{w}} \operatorname{erfc}(\tau)\right)\right\} \\
\overline{\mathbf{u}}(r, t)=\frac{2}{\sqrt{\pi}} \frac{\sqrt{c_{h} t}}{r}+\sqrt{\frac{r_{w}}{r}}\left(\frac{r-r_{w}}{r_{w}} \operatorname{erfc}(\tau)-\frac{2}{\sqrt{\pi}} \frac{\sqrt{c_{h} t}}{r_{w}} \exp \left(-\tau^{2}\right)\right)
\end{gathered}
$$

where $\tau=\frac{r-r_{w}}{2 \sqrt{c_{h} t}}$.

Two-dimensional comparison in a linear setting

To compare the SFI and ESFI procedures it was considered the same directives for the 1D setting in conjunction with data presented in Table 6 .

\begin{tabular}{|c|c|c|}
\hline Parameter & Variable [unit] & Value \\
\hline \hline Young's modulus & $E \mathrm{MPa}$ & 10000.0 \\
\hline Poisson's ratio & $\nu$ & 0.2 \\
\hline Biot's coefficient & $\alpha$ & 1 \\
\hline Fluid compressibility & $c_{f} \mathrm{MPa}^{-1}$ & 0 \\
\hline Fluid dynamic viscosity & $\eta \mathrm{Pa} \mathrm{s}^{\circ}$ & $1 \times 10^{-3}$ \\
\hline Initial porosity & $\phi^{\circ}$ & 0.1 \\
\hline Initial Abs. permeability & $\kappa^{\circ} \mathrm{m}^{2}$ & $1 \times 10^{-13}$ \\
\hline Initial hydrostatic total stress & $\sigma^{\circ} \mathrm{MPa}$ & -40 \\
\hline Initial pressure excess & $p^{\circ} \mathrm{MPa}$ & 30 \\
\hline Mud pressure & $p_{w b} \mathrm{MPa}$ & 25 \\
\hline Internal BC normal stress & $\sigma_{w b}$ & -25 \\
\hline Time step size & $\Delta t \mathrm{~s}$ & 0.0001 \\
\hline Final time & $t_{e n d} \mathrm{~s}$ & 0.01 \\
\hline Tolerance for stop criterion & $\varepsilon_{u}=\varepsilon_{p}$ & $1 \times 10^{-6}$ \\
\hline
\end{tabular}

Table 6: Parameters employed for vertical borehole test. 
Figure 9 (left and middle) shows a correct match between the early time solutions and the approximations for radial displacements and pressure along radius. It is important to point out that the early time solutions are accurate for $t \ll \frac{r_{w}^{2}}{c_{h}}$, and they should be seen as approximations for the strong solution, because that, it is opted to perform a qualitative comparison. Figure 9 (right) shows a similar effect forthcoming form the usage of ESFI for FDM, SDM, SDM ${ }^{2}$ and $S D M^{3}$ in conjunction with the mixed operator. The number of iterations per time step was decreased. The approximated solutions upcoming from SFI algorithm with low tolerances (see Table 6) demand on average 14 iterations, while the ESFI algorithm 8 iterations over the simulation time.
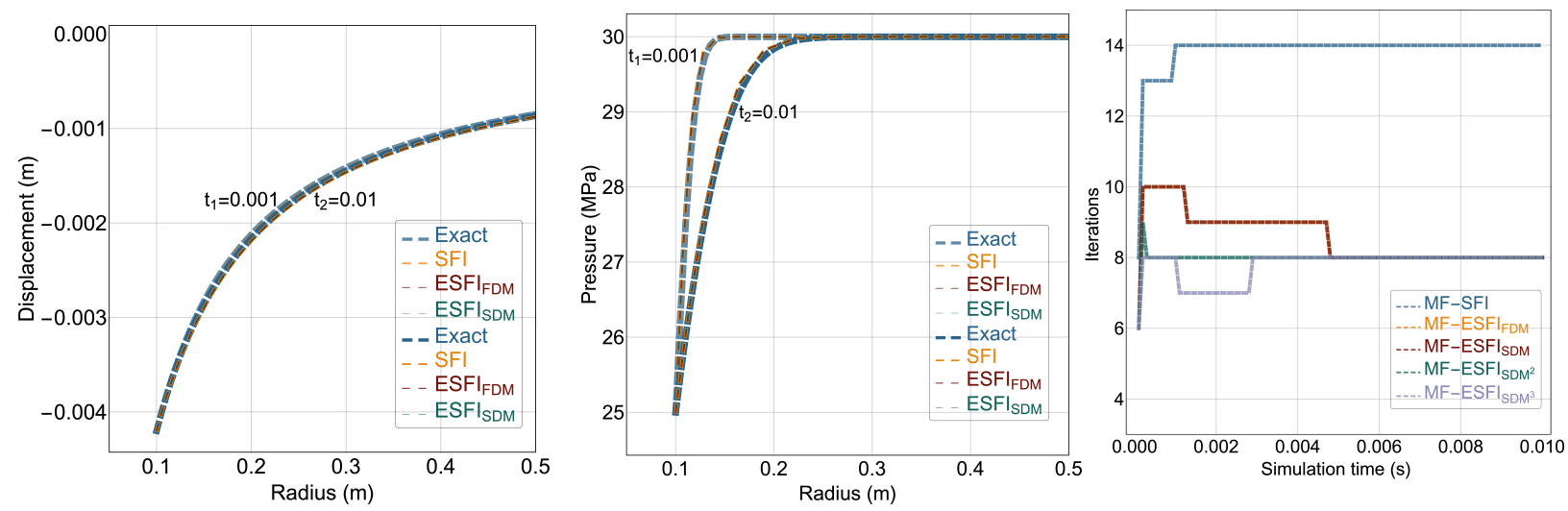

Figure 9: Plot over line for displacements (left) and pressure (middle) at two different times $t_{1}<t_{2}$. Number of iterations against simulation time for a linear two-dimensional case (right).

\subsubsection{Steady state non-linear case}

For the remaining sections, the capability of the proposed novel strategy is explored in computing approximated solutions for very challenging nonlinear problems.

\section{Two-dimensional comparison in a non-linear setting}

The physical situation considered in this subsection is similar to the linear setting, but in this case the nonlinearities arising from associative plasticity and permeability function are introduced to show the robustness for the adopted approach. The table 7 presents all the parameters used to perform the following simulations. 


\begin{tabular}{|c|c|c|}
\hline Parameter & Variable [unit] & Value \\
\hline \hline Young's modulus & $E \mathrm{MPa}$ & 5000.0 \\
\hline Poisson's ratio & $\nu$ & 0.2 \\
\hline Biot's coefficient & $\alpha$ & 1 \\
\hline Mohr-Coulomb Cohesion & $c \mathrm{MPa}$ & 8.75 \\
\hline Mohr-Coulomb Friction & $f^{\circ}$ & 30 \\
\hline Fluid compressibility & $c_{f} \mathrm{MPa}^{-1}$ & 0 \\
\hline Fluid dynamic viscosity & $\eta \mathrm{Pa} \mathrm{s}^{-3}$ & $1 \times 10^{-3}$ \\
\hline Initial porosity & $\phi^{\circ}$ & 0.1 \\
\hline Initial Abs. permeability & $\kappa^{\circ} \mathrm{m}^{2}$ & $1 \times 10^{-13}$ \\
\hline Petunin A coefficient & $A$ & $\{0,10,20,30\}$ \\
\hline Initial hydrostatic total stress & $\sigma^{\circ} \mathrm{MPa}$ & -40 \\
\hline Initial pressure excess & $p^{\circ} \mathrm{MPa}$ & 30 \\
\hline Mud pressure & $p_{w b} \mathrm{MPa}$ & 20 \\
\hline Internal BC normal stress & $\sigma_{w b}$ & -20 \\
\hline Time step size & $\Delta t \mathrm{~s}$ & 2 \\
\hline Final time & $t_{e n d} \mathrm{~s}$ & 100 \\
\hline Tolerance for stop criterion & $\varepsilon_{u}=\varepsilon_{p}$ & $1 \times 10^{-6}$ \\
\hline
\end{tabular}

Table 7: Parameters employed for vertical borehole test with nonlinearities.

All the subfigures in the figure 10 presents a comparison for the sequential approximation with an axisymmetric Runge-Kutta solver (RK), showing the verification for the implementation on the nonlinear case. A detailed description for the Runge-Kutta solver is provided on the appendix.

Figure 10 (bottom-right), presents the variation on permeability by increasing the Petunin coefficient $A$ in multiples of ten. All the quantities are reported along the red line presented by the miniature figure in effective stress plot (top-right). Considering plane strain conditions, near to the wellbore can be appreciated an increment on permeability due to the positive radial total strain with is bigger than the azimuthal total strain (see figure 10 (bottom-left) ). These two values have different sign and magnitude near to the wellbore leading to a positive total volumetric strain. Because of pressure data is prescribed on boundaries, the pressure (middle-left) distribution along the radius is almost the same, thus the change in permeability leads to a change in the flux magnitude, consequently, the quantity of mass extracted from the system is decreased leading to subtle changes in the other quantities effective stress (top-right) and displacement (top-left), presented in figure 10. 

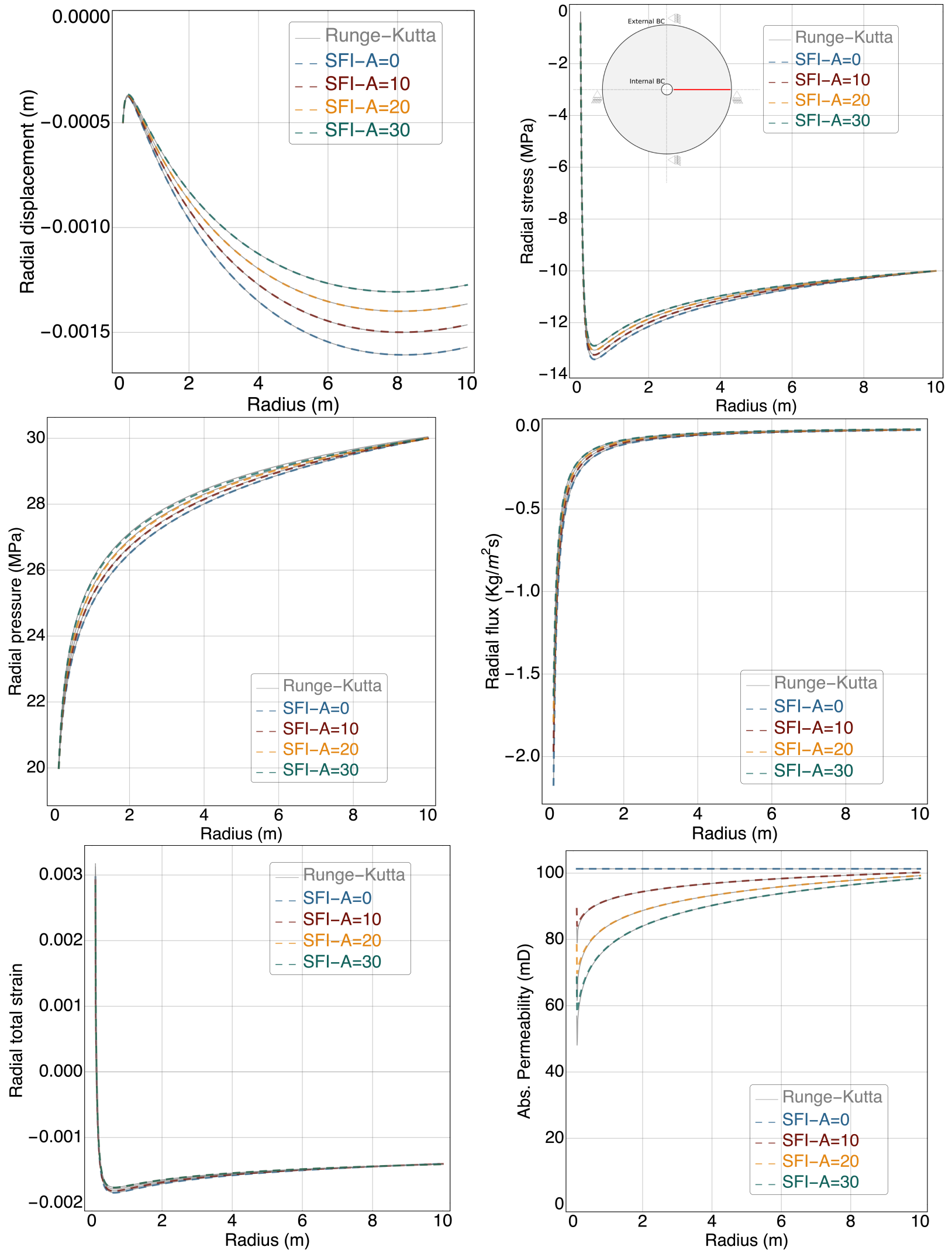

Figure 10: Nonlinear two-dimensional case: steady state approximation for several values of $A=\{0,10,20,30\}$. Displacement (top-left), effective stress (top-right), pressure (middle-left), flux (middle-right), total strain (bottomleft), and absolute permeability (bottom-right). 


\subsection{Verification for the ESFI under poroelastoplastic deformation}

To compare the ESFI and FC procedures it was considered the same directives for the twodimensional case in a nonlinear setting in conjunction with data presented in Table 8 .

\begin{tabular}{|c|c|c|}
\hline Parameter & Variable [unit] & Value \\
\hline \hline Young's modulus & $E \mathrm{MPa}$ & 5000.0 \\
\hline Poisson's ratio & $\nu$ & 0.2 \\
\hline Biot's coefficient & $\alpha$ & 0.815 \\
\hline Mohr-Coulomb Cohesion & $c \mathrm{MPa}$ & 6.75 \\
\hline Mohr-Coulomb Friction & $f^{\circ}$ & 25 \\
\hline Fluid compressibility & $c_{f} \mathrm{MPa}^{-1}$ & 0 \\
\hline Fluid dynamic viscosity & $\eta \mathrm{Pa} \mathrm{s}^{-3}$ & $1 \times 10^{-3}$ \\
\hline Initial porosity & $\phi^{\circ}$ & 0.1 \\
\hline Initial Abs. permeability & $\kappa^{\circ} \mathrm{m}^{2}$ & $1 \times 10^{-13}$ \\
\hline Petunin A coefficient & $A$ & 20 \\
\hline Initial hydrostatic total stress & $\sigma^{\circ} \mathrm{MPa}$ & -40 \\
\hline Initial pressure excess & $p^{\circ} \mathrm{MPa}$ & 30 \\
\hline Mud pressure & $p_{w b} \mathrm{MPa}$ & 20 \\
\hline Internal BC normal stress & $\sigma_{w b}$ & -20 \\
\hline Time step size & $\Delta t \mathrm{~s}$ & 2 \\
\hline Final time & $t_{e n d} \mathrm{~s}$ & 100 \\
\hline Tolerance for stop criterion & $\varepsilon_{u}=\varepsilon_{p}$ & $1 \times 10^{-6}$ \\
\hline
\end{tabular}

Table 8: Parameters employed for vertical borehole ESFI verification with nonlinearities.

Figure 11 (top-left) shows the variation of volumetric plastic strain near wellbore rendered as vertical warp by scalar at time value 30.0 [s]. Figure 11 (top-right) displays the variation of the modified Biot's coefficient around the wellbore. It was rendered as vertical warp by scalar, and it documents the discontinuous behavior of the modified Biot's coefficient. da Silva et al. [59] state that as volumetric plastic strain increase, the modified Biot coefficient $\alpha$ trends to 1.0. Figure 11 (top-right) verifies this fact showing a change from 0.815 to 0.849 near to the wellbore at time value 30.0 [s]. Figure 11 (bottom-left and bottom-right) presents a correct match for the approximations for pressure and radial effective stress along radius, at two different time values. Figure 11 documents the validity of the proposed ESFI scheme during approximating a poroelastoplastic solution. 

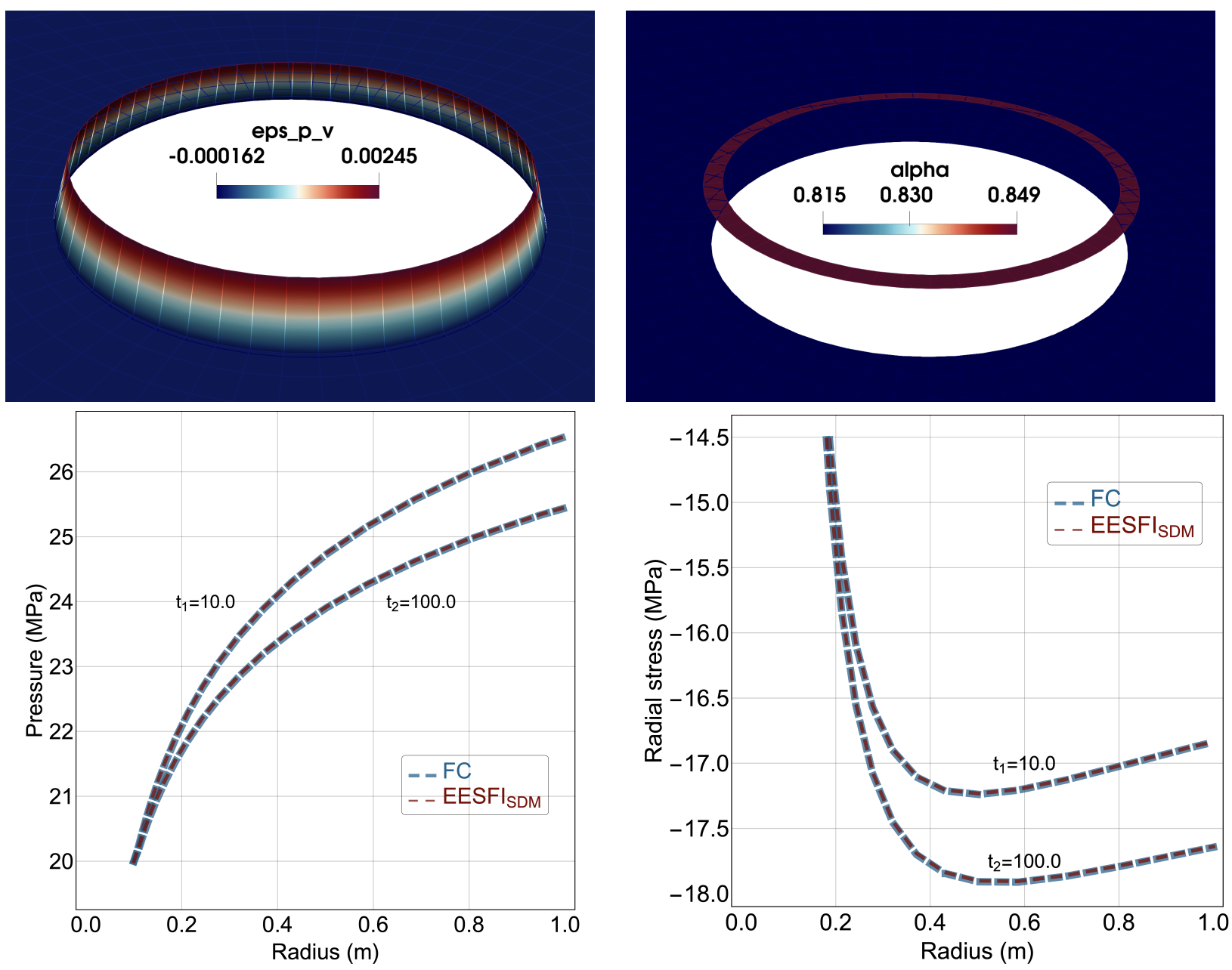

Figure 11: Nonlinear two-dimensional case: Transient approximation for $A=20$. Volumetric plastic strain rendered as warp by scalar (top-left), modified Biot's coefficient rendered as warp by scalar (top-right), pressure (bottom-left), and radial effective stress (bottom-right).

\subsubsection{Transient non-linear case}

In this subsection a transient nonlinear simulation was performed considering $A=20$ and the parameters presented in table 7 . The physical conditions are similar to the steady-state described previously. 

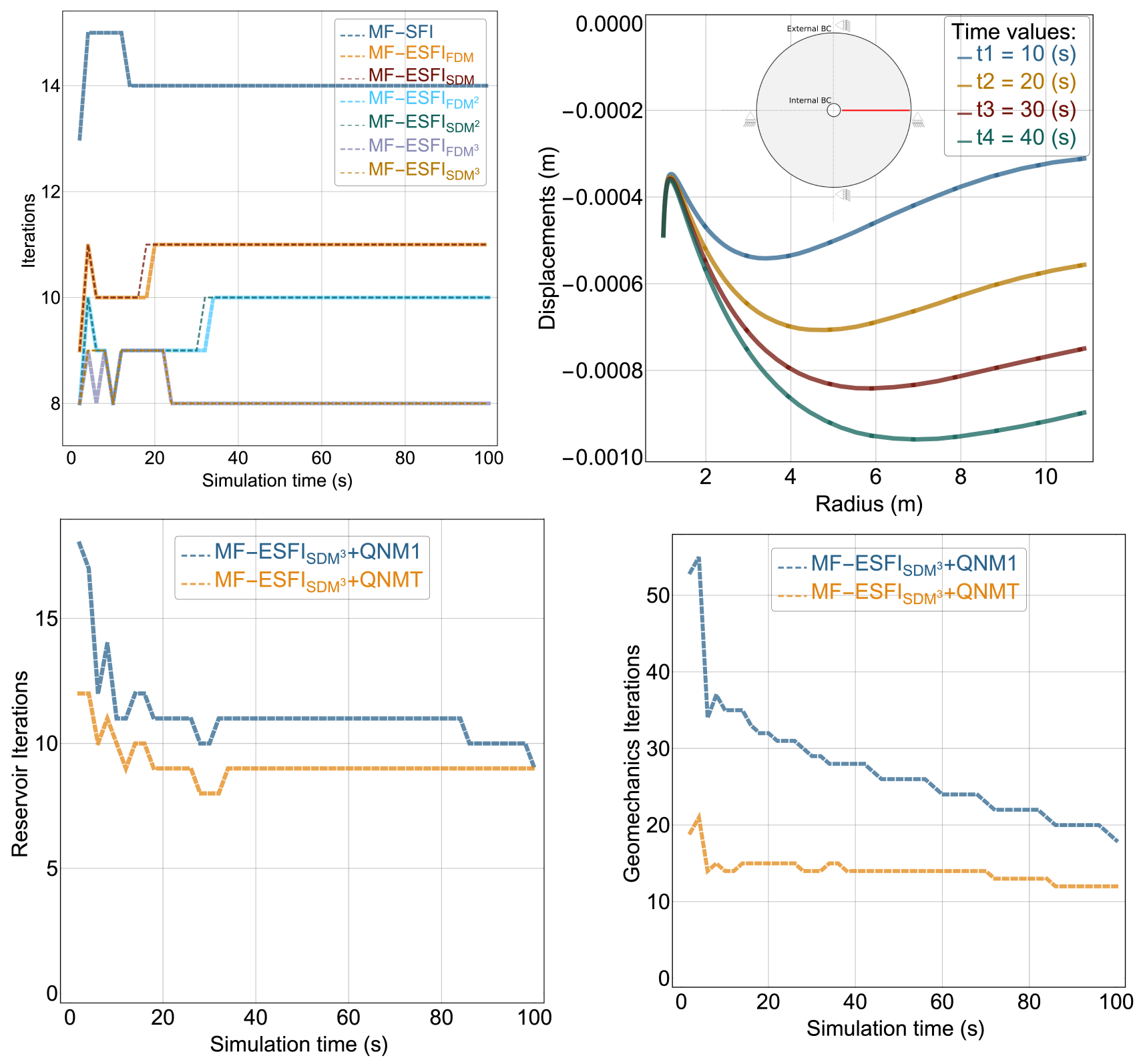

Figure 12: Nonlinear two-dimensional case: Number of external loop iterations against simulation time (top-right), and displacement evolution at $t=\{10,20,30,40\}$ for $A=20$ (top-right). Internal iterations per sequential process for reservoir equations (bottom-left) and geomechanic equations (bottom-right).

The figure 12 presents several features that are going to be commented as follows:

1. On the left the number of iterations for the SFI and ESFI are obtained for several versions of accelerations separated by the terms $F D M$ and $S D M$, and by the number of recursions in the sense of Shanks transformations. As is documented by several experiments in [73] there is not any kind of supremacy related to $F D M$ and $S D M$, and their number of iterations is equal for all the cases. It was observed also for linear cases. The effect for the recursion formula, in any case, shows that there is a gain in efficiency respect to the SFI. In average the number for iterations was reduced by $25 \%$ when 3 states are accessible. There is an extra reduction to $33.33 \%$ when the recursion is used and there are 5 states available. For an extra 
recursion, there is a small gain with using 7 states or more. It implies that when the stop criterion is less tight, i.e. larger tolerances, the most suitable method corresponds to do not using recursion, but when the number of iterations are convenient, the use of the recursion provides the a most efficient approximation;

2. On the right, the radial displacement evolution is shown for several time values $t=\{10,20,30,40\}[\mathrm{s}]$. In terms of number of iterations, the best gain in reduction is attributed to the first time values $t=\{10,20,30\} \quad[\mathrm{s}]$ when the solution change dramatically, and the effect for the recursions are more evident. From the time value $t=40[\mathrm{~s}]$ to final one $t_{\text {end }}=100[\mathrm{~s}]$, the changes in the state variables are subtle, and as a consequence the number of iterations became constant;

3. The figures at the bottom shows a comparison when the internal acceleration applied to each subproblem is applied. The number of iterations required to attain the stop criteria during the nonlinear solver are reduced, when the internal acceleration $\mathrm{QNM}_{T}$ is applied for each subproblem, instead of the unmodified approach $\mathrm{QNM}_{1}$.

\subsection{Drilling of a borehole in a three-dimensional reservoir}

In this subsection the simulation of a $3 \mathrm{D}$ wellbore region with similar physical setting to the nonlinear 2D is presented. The parameters are presented in table 9 and two different vertical total stress $\sigma_{z z}^{\circ}$ values standing for hydrostatic initial data (Case 1) and compaction scenario (Case 2).

\begin{tabular}{|c|c|c|}
\hline Parameter & Variable [unit] & Value \\
\hline \hline Reservoir thickness & $\mathrm{h}_{r e s} \mathrm{~m}$ & 5.0 \\
\hline \hline Young's modulus & $E \mathrm{MPa}$ & 5000.0 \\
\hline Poisson's ratio & $\nu$ & 0.2 \\
\hline Biot's coefficient & $\alpha$ & 1 \\
\hline Mohr-Coulomb Cohesion & $c \mathrm{MPa}$ & 6.75 \\
\hline Mohr-Coulomb Friction & $f^{\circ}$ & 25 \\
\hline Fluid compressibility & $c_{f} \mathrm{MPa}^{-1}$ & 0 \\
\hline Fluid dynamic viscosity & $\eta \mathrm{Pa} \mathrm{s}^{\mathrm{s}}$ & $1 \times 10^{-3}$ \\
\hline Initial porosity & $\phi^{\circ}$ & 0.1 \\
\hline Initial Abs. permeability & $\kappa^{\circ} \mathrm{m}^{2}$ & $1 \times 10^{-13}$ \\
\hline Petunin A coefficient & $A$ & 20 \\
\hline Initial horizontal total stress & $\sigma_{r r}^{\circ}=\sigma_{\theta \theta}^{\circ} \mathrm{MPa}$ & -40 \\
\hline Case 1: Initial vertical total stress & $\sigma_{z z}^{\circ} \mathrm{MPa}$ & -40 \\
\hline Case 2: Initial vertical total stress & $\sigma_{z z}^{\circ} \mathrm{MPa}$ & -80 \\
\hline Initial pressure excess & $p^{\circ} \mathrm{MPa}$ & 30 \\
\hline Fluid pressure & $p_{w b} \mathrm{MPa}$ & 20 \\
\hline Internal BC normal stress & $\sigma_{w b}$ & -20 \\
\hline Time step size & $\Delta t \mathrm{~s}$ & 2 \\
\hline Final time & $t_{e n d} \mathrm{~s}$ & 100 \\
\hline Tolerance for stop criterion & $\varepsilon_{u}=\varepsilon_{p}$ & $1 \times 10^{-6}$ \\
\hline
\end{tabular}

Table 9: Parameters employed for vertical borehole 3D with nonlinearities.

All the plots presented in figure 13 are reported for two different time values $t=\{10,100\}[\mathrm{s}]$. The figure at middle-right position presents the permeability change due to pressure drop while 
vertical total stress $\sigma_{z z}^{\circ}$ is kept constant along the simulation time. In the previous 2D case under plane strain, there was observed an increase of permeability due to the fact that vertical compaction is not venerated, it can be probably controlled by plane stress assumption but it is out of the scope of this research. Considering the third dimension allows easily to venerate the vertical compaction and the permeability always decreases over the entire wellbore wall. This characteristic is observed when the permeability variation of the hydrostatic compaction (Case 1) is compared to the vertical compaction (Case 2). The figure at top-right position shows the radial flux for both cases. The pressure data is prescribed at internal and external boundaries, as a consequence the radial flux decrease as the permeability decrease. The figure at middle-left position documents the effect for the augmented Shanks acceleration proposed in this research allowing us to obtain 3D dimensional approximations more efficiently when is used only SFI as a sequential solver. The figure at top-left position displays the effect of the vertical compaction on the subsidence triggered by the reservoir pressure change. The plot is rendered at the reservoir top. As it is expected the vertical compaction case shows large subsidence values in comparison with the hydrostatic compaction case. The figure at bottom-left position presents the geometrical partition used for the simulations, it is composed as a structured mesh with second order hexahera for better geometrical representation. The figure at bottom-right shows the critical region where the permeability suffers at least a reduction of 10 $\%$. It can be observed that from only geomechanical effects the wellbore region deteriorates the productivity index associated with the case when is considered a constant permeability and no geomechanic effects. 

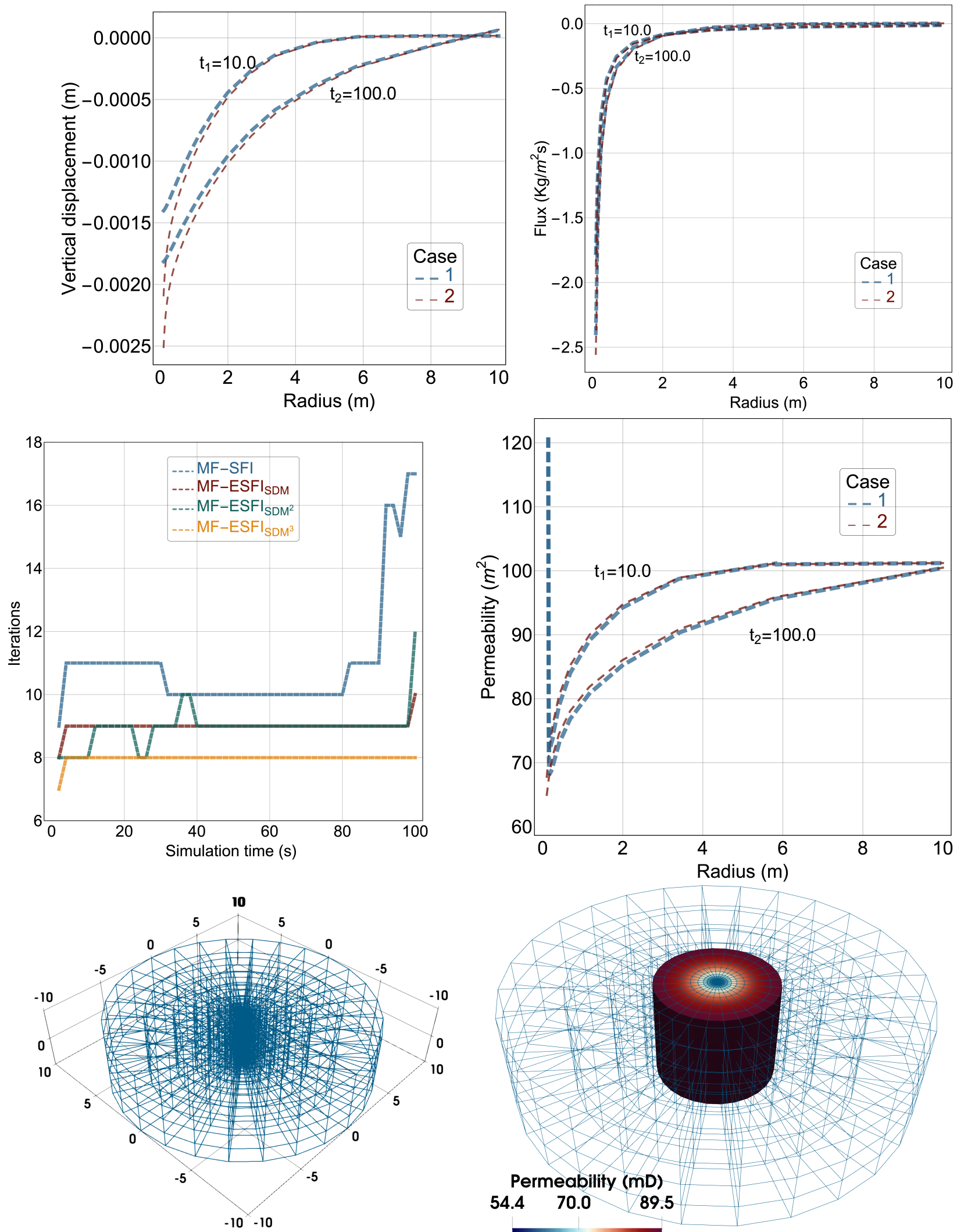

Figure 13: Nonlinear three-dimensional case: Transient approximation for $A=20$. Vertical displacement at wellbore top (top-left), radial flux (top-right), number of iterations (middle-left), permeability (middle-right), geometry hexahedral partition with quadratic elements (bottom-left), and region with at least $10 \%$ of reduction permeability (bottom-right). 


\section{Conclusions}

An enhanced version for a sequential fully implicit ESFI has been proposed. It combines several nonlinear accelerations techniques at the level of external and internal loops to obtain an accelerated convergence. A systematic procedure for the construction of the acceleration of the external loop is developed considering the recursion of known acceleration formulas in the sense of Shanks transformations. Furthermore, a Runge-Kutta axisymmetric solver for poroelastoplasticity is presented as a manner to verify the implemented equations. Important characteristics are, ESFI can be applied to generate approximations of several linear and nonlinear constitutive models in 1D, 2D, and 3D simulations, it makes use of a reduced number of iterations to approximated several challenging problems forthcoming reservoir geomechanics applications, and it can be extended to several multiphysics solvers that make use of split operators and execute them in a sequential manner.

\section{Acknowledgements}

The authors O. Duran, M. Sanei, and P.R.B. Devloo thankfully acknowledge financial support from the Brazilian National Agency of Petroleum, Natural Gas and Biofuels (ANP-PETROBRAS).

\section{Appendix}

A Runge-Kutta solver for poroelastoplasticity: An asymmetrical approach

The linear poroelastic case

To construct an Runge-Kutta approximation it is required to review the poroelastic equations and find a way to recast the equations as initial value problem, as the Runge-Kutta method structure:

$$
\frac{d \mathbf{y}}{d \mathbf{x}}=\mathbf{f}(\mathbf{y})
$$

There are three main considerations for this case:

1. The equations are presented in terms of the Cylindrical coordinate system.

2. The approximation is axisymmetric leading to a displacement $\mathbf{u}$ and pressure $p$ fields that depends only of the radius, i.e. $\mathbf{u}=\Phi(r)$ and $p=\Phi(r)$.

3. Assume steady state conditions, the initial value problem is described in terms of one independent variable $r$.

Recalling the linear elastic constitutive law:

$$
\boldsymbol{\sigma}=2 \mu\left(\boldsymbol{\epsilon}-\boldsymbol{\epsilon}^{\circ}\right)+\lambda \operatorname{tr}\left(\boldsymbol{\epsilon}-\boldsymbol{\epsilon}^{\circ}\right) \mathbf{I}-\sigma^{\circ} \mathbf{I} \text { and } \boldsymbol{\epsilon}=\frac{1}{2}\left(\nabla \mathbf{u}+\nabla^{T} \mathbf{u}\right)
$$

Using the considerations above $\mathbf{u}=u_{r} \hat{\mathbf{r}}$ and an initial $\boldsymbol{\epsilon}^{\circ}=0$ the effective stress tensor becomes:

$$
\boldsymbol{\sigma}=\left(\mu_{r}+\lambda_{r}\right) \hat{\mathbf{r}} \otimes \hat{\mathbf{r}}+\left(\mu_{r}+\lambda_{r}\right) \hat{\boldsymbol{\theta}} \otimes \hat{\boldsymbol{\theta}}+\left(\lambda_{r}\right) \hat{\mathbf{z}} \otimes \hat{\mathbf{z}}
$$

where $\mu_{r}=2 \mu \frac{d u_{r}}{d r}$ and $\lambda_{r}=\lambda\left(\frac{u_{r}}{r}+\frac{d u_{r}}{d r}\right)$. Taking the trace of the expression above can be obtained the following expression for $\frac{d u_{r}}{d r}$ : 


$$
\frac{d u_{r}}{d r}=\frac{r \sigma_{r r}-\lambda u_{r}}{r(\lambda+2 \mu)}
$$

Evoke the total stress $\boldsymbol{\sigma}_{t}$ equilibrium and using the Biot decomposition of the total stress:

$$
\operatorname{div}\left(\boldsymbol{\sigma}-\sigma^{\circ} \mathbf{I}-\alpha\left(p-p^{\circ}\right) \mathbf{I}\right)=0
$$

The expression for $\frac{d \sigma_{r r}}{d r}$ is obtained from the momentum conservation directly:

$$
\frac{d \sigma_{r r}}{d r}=\frac{-\sigma_{r r}+\left(\frac{2 \mu u_{r}}{r}+\lambda\left(\frac{u_{r}}{r}+\frac{r \sigma_{r r}-\lambda u_{r}}{r(\lambda+2 \mu)}\right)\right)}{r}-\alpha \frac{\eta}{k} q_{r}
$$

Also, the quantities $\sigma_{\theta \theta}$ and $\sigma_{z z}$ are:

$$
\begin{gathered}
\sigma_{\theta \theta}=\frac{2 \mu u_{r}}{r}+\lambda\left(\frac{u_{r}}{r}+\frac{r \sigma_{r r}-\lambda u_{r}}{r(\lambda+2 \mu)}\right) \\
\sigma_{z z}=\frac{\lambda\left(\lambda\left(\sigma_{r r}+\sigma_{\theta \theta}\right)+2 \mu\left(\sigma_{r r}+\sigma_{\theta \theta}\right)\right)}{2(\lambda+\mu)(\lambda+2 \mu)}
\end{gathered}
$$

Reinstate that Darcy constitutive expression provides the expression for $\frac{d p}{d r}$ :

$$
\frac{d p}{d r}=-\frac{\eta}{\kappa} q_{r}
$$

Finally, the mass conservation equation affords the expression for $\frac{d q_{r}}{d r}$ :

$$
\frac{d q_{r}}{d r}=-\frac{q_{r}}{r}
$$

Regarding to the initial value problem the spatial derivative for variable $\mathbf{y}$ is clearly:

$$
\frac{d \mathbf{y}}{d \mathbf{x}}=\left\{\begin{array}{c}
\frac{d u_{r}}{d r} \\
\frac{d \sigma_{r r}}{d r} \\
\frac{d p}{d r} \\
\frac{d q_{r}}{d r}
\end{array}\right\} \text { and } \mathbf{f}(\mathbf{y})=\left\{\begin{array}{c}
\frac{r \sigma_{r r}-\lambda u_{r}}{r(\lambda+2 \mu)} \\
\frac{-\sigma_{r r}+\left(\frac{2 \mu u_{r}}{r}+\lambda\left(\frac{u_{r}}{r}+\frac{r \sigma_{r r}-\lambda u_{r}}{r(\lambda+2 \mu)}\right)\right)}{r}-\alpha \frac{\eta}{k} q_{r} \\
-\frac{\eta}{\kappa} q_{r} \\
-\frac{q_{r}}{r}
\end{array}\right\}
$$

For the completeness of the initial value problem the data $\mathbf{y}^{\circ}$ is evaluated at the permeability reservoir radius $r_{e}$. It is important to point that the permeability can be one function of the state variable $\mathbf{y}$ in a nonlinear sense.

The poroelastoplastic case

The expression (87) can be rewritten in terms of strain and stress data: 


$$
\frac{d \mathbf{y}}{d \mathbf{x}}=\left\{\begin{array}{c}
\frac{d u_{r}}{d r} \\
\frac{d \sigma_{r r}}{d r} \\
\frac{d p}{d r} \\
\frac{d q_{r}}{d r}
\end{array}\right\} \text { and } \mathbf{f}(\mathbf{y})=\left\{\begin{array}{c}
\epsilon_{r r} \\
\frac{-\sigma_{r r}+\sigma_{\theta \theta}}{r}-\alpha \frac{\eta}{k} q_{r} \\
-\frac{\eta}{\kappa_{q_{r}}} q_{r} \\
-\frac{q_{r}}{r}
\end{array}\right\}
$$

Thus, the approximation above can be recast as elastoplastic problem by delaying $\alpha, K_{d r}$, and the elastoplastic strain between two consecutive points in order to consider the nonlinear effects of plasticity during the RK process. The pay off is a very similar implementation for the RK solver with the need of additional discrete points to reach a reasonable approximation.

For the completeness of the initial value problem the data $\mathbf{y}^{\circ}$ is evaluated at the permeability reservoir radius $r_{e}$ where it is assumed to be linear poroelastic data. For that reason, it is expected that as the number of discrete points increase the poroelastoplastic approximation accuracy increase. To conclude, the RK solver is a suitable scheme for approximation and/or verification purposes.

\section{References}

[1] P. J. Phillips, M. F. Wheeler, A coupling of mixed and continuous galerkin finite element methods for poroelasticity i: the continuous in time case, Computational Geosciences 11 (2007) 131-144.

[2] H. Merle, C. Kentie, G. van Opstal, G. Schneider, The bachaquero study - a composite analysis of the behavior of a compaction drive/solution gas drive reservoir, Journal of Petroleum Technology 28 (1976) 1107-1115.

[3] D. Kosloff, R. Scott, J. Scranton, Finite element simulation of wilmington oil field subsidence: I. linear modelling, Tectonophysics 65 (1980) 339-368.

[4] P. H. Winterfeld, Y. Wu, Coupled reservoir-geomechanical simulation of caprock failure and fault reactivation during $\mathrm{CO}_{2}$ sequestration in deep saline aquifers, Paper SPE-182605-MS presented at the SPE Reservoir Simulation Conference, 20-22 February, Montgomery, Texas, USA (2017).

[5] M. Bruno, Subsidence-induced well failure, SPE Drilling Engineering 7 (1992) 148-152.

[6] J. Davies, D. Davies, Stress-dependent permeability: Characterization and modeling, SPE Journal 6 (2001) 224-235.

[7] B. Ju, Y. Wu, T. Fan, Study on fluid flow in nonlinear elastic porous media: Experimental and modeling approaches, Journal of Petroleum Science and Engineering 76 (2011) 205-211.

[8] K. Terzaghi, Erdbaumechanik auf bodenphysikalischer grundlage, Franz Deutikle, Leipzig und Wien, 1925.

[9] M. A. Biot, General theory of three-dimensional consolidation, Journal of Applied Physics 12 (1941) 155-164. 
[10] A. Settari, F. Mourits, A coupled reservoir and geomechanical simulation system, SPE Journal 3 (1998) 219-226.

[11] D. Yale, S. Lyons, G. Qin, Coupled geomechanics-fluid flow modeling in petroleum reservoirs: Coupled versus uncoupled response, 4th North American Rock Mechanics Symposium, Seattle, Washington (2000).

[12] P. J. Phillips, M. F. Wheeler, A coupling of mixed and continuous galerkin finite element methods for poroelasticity II: the discrete-in-time case, Computational Geosciences 11 (2007) $145-158$.

[13] T. Dung, Coupled fluid flow-geomechanics simulations applied to compaction and subsidence estimation in stress sensitive and heterogeneous reservoirs, 2007.

[14] Z. Wei, D. Zhang, Coupled fluid-flow and geomechanics for triple-porosity/dual-permeability modeling of coalbed methane recovery, International Journal of Rock Mechanics and Mining Sciences 47 (2010) 1242-1253.

[15] S. Peng, J. Zhang, Stress-dependent permeability, in: Engineering Geology for Underground Rocks, Springer Berlin Heidelberg, Berlin, Heidelberg, 2007, pp. 199-220.

[16] U. Aybar, M. O. Eshkalak, K. Sepehrnoori, T. Patzek, Long term effect of natural fractures closure on gas production from unconventional reservoirs, Paper SPE-171010-MS presented at the SPE Eastern Regional Meeting, 21-23 October, Charleston, WV, USA (2014).

[17] C. An, J. Killough, L. Mi, Stress-dependent permeability of organic-rich shale reservoirs: Impacts of stress changes and matrix shrinkage, Journal of Petroleum Science and Engineering 172 (2019) $1034-1047$.

[18] M. S. Gutierrez, R. W. Lewis, Coupling of fluid flow and deformation in underground formations, Journal of Engineering Mechanics 128 (2002) 779-787.

[19] X. Li, Z. Liu, R. W. Lewis, Mixed finite element method for coupled thermo-hydro-mechanical process in poro-elasto-plastic media at large strains, International Journal for Numerical Methods in Engineering 64 (2005) 667-708.

[20] B. Jha, R. Juanes, A locally conservative finite element framework for the simulation of coupled flow and reservoir geomechanics, Acta Geotechnica 2 (2007) 139-153.

[21] S. E. Minkoff, C. M. Stone, S. Bryant, M. Peszynska, M. F. Wheeler, Coupled fluid flow and geomechanical deformation modeling, Journal of Petroleum Science and Engineering 38 (2003) $37-56$.

[22] F. Armero, J. C. Simo, A new unconditionally stable fractional step method for non-linear coupled thermomechanical problems, International Journal for Numerical Methods in Engineering 35 (1992) 737-766.

[23] R. H. Dean, X. Gai, C. M. Stone, S. E. Minkoff, A comparison of techniques for coupling porous flow and geomechanics, SPE Journal 11 (2006) 132-140.

[24] M. F. Wheeler, X. Gai, Iteratively coupled mixed and galerkin finite element methods for poro-elasticity, Numerical Methods for Partial Differential Equations 23 (2007) 785-797. 
[25] S. Dana, B. Ganis, M. F. Wheeler, A multiscale fixed stress split iterative scheme for coupled flow and poromechanics in deep subsurface reservoirs, Journal of Computational Physics 352 (2018) 1-22.

[26] J. Kim, H. Tchelepi, R. Juanes, Stability and convergence of sequential methods for coupled flow and geomechanics: Drained and undrained splits, Computer Methods in Applied Mechanics and Engineering 200 (2011) 2094-2116.

[27] J. Kim, H. A. Tchelepi, R. Juanes, Stability and convergence of sequential methods for coupled flow and geomechanics: Fixed-stress and fixed-strain splits, Computer Methods in Applied Mechanics and Engineering 200 (2011) 1591-1606.

[28] J. Kim, H. A. Tchelepi, R. Juanes, Stability, accuracy, and efficiency of sequential methods for coupled flow and geomechanics, SPE Journal 16 (2011) 249-262.

[29] A. Mikelić, M. F. Wheeler, Convergence of iterative coupling for coupled flow and geomechanics, Computational Geosciences 17 (2012) 455-461.

[30] J. Jiang, H. A. Tchelepi, Nonlinear acceleration of sequential fully implicit (sfi) method for coupled flow and transport in porous media, Computer Methods in Applied Mechanics and Engineering 352 (2019) $246-275$.

[31] A. Aitken, Studies in practical mathematics. the evaluation of latent roots and latent vectors of a matrix, Proceedings of the Royal Society of Edinburgh 57 (1937) 269.

[32] M. Garbey, Acceleration of the schwarz method for elliptic problems, SIAM Journal on Scientific Computing 26 (2005) 1871-1893.

[33] A. Jemcov, J. Maruszewski, H. Jasak, Acceleration and Stabilization of Algebraic Multigrid Solver Applied to Incompressible Flow Problems, p. 4330.

[34] J. Degroote, K.-J. Bathe, J. Vierendeels, Performance of a new partitioned procedure versus a monolithic procedure in fluid-structure interaction, Computers \& Structures 87 (2009) 793-801.

[35] P. Erbts, A. Düster, Accelerated staggered coupling schemes for problems of thermoelasticity at finite strains, Computers \& Mathematics with Applications 64 (2012) 2408-2430.

[36] A. Aitken, On bernoulli's numerical solution of algebraic equations, Proceedings of the Royal Society of Edinburgh 46 (1926) 289-305.

[37] D. Plancq, G. Thouvenin, J. Ricaud, C. Struzik, T. Helfer, F. Bentejac, P. Thévenin, R. Masson, Pleiades: a unified environment for multi-dimensional fuel performance modeling, International meeting on LWR fuel performance, Florida (2004).

[38] A. Jennings, Accelerating the convergence of matrix iterative processes, IMA Journal of Applied Mathematics 8 (1971) 99-110.

[39] L. F. Richardson, The approximate arithmetical solution by finite differences of physical problems involving differential equations, with an application to the stresses in a masonry dam, Philosophical Transactions of the Royal Society A: Mathematical, Physical and Engineering Sciences 210 (1911) 307-357. 
[40] D. Shanks, Non-linear transformations of divergent and slowly convergent sequences, Journal of Mathematics and Physics 34 (1955) 1-42.

[41] V. V. Petunin, A. N. Tutuncu, M. Prasad, H. Kazemi, X. Yin, An experimental study for investigating the stress dependence of permeability in sandstones and carbonates, 45th U.S. Rock Mechanics / Geomechanics Symposium 9 (2011).

[42] E. A. de Souza Neto, D. Peri, D. R. J. Owen, Computational Methods for Plasticity, John Wiley Sons, Ltd, 2008.

[43] P. R. B. Devloo, Object oriented tools for scientific computing, Engineering with Computers 16 (2000) 63-72.

[44] P. R. B. Devloo, C. M. A. A. Bravo, E. C. Rylo, Systematic and generic construction of shape functions for p-adaptive meshes of multidimensional finite elements, Computer Methods in Applied Mechanics and Engineering 198 (2009) 1716-1725.

[45] A. M. Farias, New formulations of finite element and multiphysics simulation, Ph.D. thesis, University State of Campinas, 2014.

[46] D. A. Castro, P. R. Devloo, A. M. Farias, S. M. Gomes, D. de Siqueira, O. Duran, Three dimensional hierarchical mixed finite element approximations with enhanced primal variable accuracy, Computer Methods in Applied Mechanics and Engineering 306 (2016) 479-502.

[47] K. Terzaghi, Theoretical Soil Mechanics, John Wiley \& Sons, Inc., 1943.

[48] M. A. Biot, Theory of elasticity and consolidation for a porous anisotropic solid, Journal of Applied Physics 26 (1955) 182-185.

[49] O. Coussy, Poromechanics, Wiley, 2004.

[50] J. W. Rudnicki, Fluid mass sources and point forces in linear elastic diffusive solids, Mechanics of Materials 5 (1986) 383-393.

[51] D. L. Cecílio, P. R. Devloo, S. M. Gomes, E. R. S. Santos, N. Shauer, An improved numerical integration algorithm for elastoplastic constitutive equations, Computers and Geotechnics 64 (2015) 1-9.

[52] K. Hayakawa, S. Murakami, Y. Liu, An irreversible thermodynamics theory for elastic-plasticdamage materials, European Journal of Mechanics - A/Solids 17 (1998) 13-32.

[53] J. Lemaitre, J.-L. Chaboche, Mechanics of Solid Materials, Cambridge University Press, 1990.

[54] A. C. Soares, Um estudo da Influéncia do Estado de Tensões na Permeabilidade de Rochas Produtoras de Petróleo. Tese de Doutorado, Instituto de Geologia, Ph.D. thesis, Universidade Federal do Rio de Janeiro, 2007.

[55] E. S. R. Santos, A. M. Borba, F. H. Ferreira, Stress-dependent permeability measurement of indiana limestone and silurian dolomite samples in hydrostatic tests, International Society for Rock Mechanics and Rock Engineering, Goiania, Brazil 04 (2014). 
[56] E. Detournay, A. H.-D. Cheng, Fundamentals of poroelasticity, in: J. A. Hudson (Ed.), Comprehensive Rock Comprehensive Rock Engineering: Principles, Practice \& Projects, Analysis and Design Methods, Oxford University Press, Oxford, 1993, pp. 113-171.

[57] T. A. Bui, H. Wong, F. Deleruyelle, A. Zhou, X. Lei, A coupled poroplastic damage model accounting for cracking effects on both hydraulic and mechanical properties of unsaturated media, International Journal for Numerical and Analytical Methods in Geomechanics 40 (2016) 625-650.

[58] H. Zhou, Y. Jia, J. Shao, A unified elastic-plastic and viscoplastic damage model for quasibrittle rocks, International Journal of Rock Mechanics and Mining Sciences 45 (2008) 1237 1251.

[59] R. O. S. da Silva, M. A. M. Murad, J. A. L. O. Obregon, A new fixed-stress split scheme in poroplastic media incorporating general plastic porosity constitutive theories, in: ECMOR XVI - 16th European Conference on the Mathematics of Oil Recovery, EAGE Publications BV, 2018, pp. 1-25.

[60] S. Y. Xie, J. F. Shao, An experimental study and constitutive modeling of saturated porous rocks, Rock Mechanics and Rock Engineering 48 (2015) 223-234.

[61] T. Ertekin, Basic Applied Reservoir Simulation, Society of Petroleum Engineers, 2001.

[62] J. Kim, , E. L. Sonnenthal, J. Rutqvist, Formulation and sequential numerical algorithms of coupled fluid/heat flow and geomechanics for multiple porosity materials, International Journal for Numerical Methods in Engineering 92 (2012) 425-456.

[63] J. W. Both, K. Kumar, J. M. Nordbotten, F. A. Radu, Anderson accelerated fixed-stress splitting schemes for consolidation of unsaturated porous media, Computers \& Mathematics with Applications 77 (2019) 1479 - 1502. 7th International Conference on Advanced Computational Methods in Engineering (ACOMEN 2017).

[64] D. Boffi, F. Brezzi, M. Fortin, Mixed Finite Element Methods and Applications, Springer Berlin Heidelberg, 2013.

[65] N. Castelletto, S. Klevtsov, H. Hajibeygi, H. A. Tchelepi, Multiscale two-stage solver for biot's poroelasticity equations in subsurface media, Computational Geosciences 23 (2019) 207-224.

[66] J. A. White, N. Castelletto, S. Klevtsov, Q. M. Bui, D. O.-K., H. A. Tchelepi, A two-stage preconditioner for multiphase poromechanics in reservoir simulation, Computer Methods in Applied Mechanics and Engineering 357 (2019) 112575.

[67] S. W. Sloan, D. Sheng, A. J. Abbo, Accelerated initial stiffness schemes for elastoplasticity, International Journal for Numerical and Analytical Methods in Geomechanics 24 (2000) 579599 .

[68] A. Cordero, J. L. Hueso, E. M., J. R. Torregrosa, Increasing the convergence order of an iterative method for nonlinear systems, Applied Mathematics Letters 25 (2012) 2369 - 2374.

[69] X.-Y. Xiao, H.-W. Yin, Accelerating the convergence speed of iterative methods for solving nonlinear systems, Applied Mathematics and Computation 333 (2018) 8-19. 
[70] J. Degroote, R. Haelterman, S. Annerel, P. Bruggeman, J. Vierendeels, Performance of partitioned procedures in fluid-structure interaction, Computers \& Structures 88 (2010) 446-457.

[71] A. E. J. Bogaers, S. Kok, B. D. Reddy, T. Franz, An evaluation of quasi-newton methods for application to FSI problems involving free surface flow and solid body contact, Computers \& Structures 173 (2016) 71-83.

[72] T. Liu, S. Bouaziz, L. Kavan, Quasi-newton methods for real-time simulation of hyperelastic materials, ACM Transactions on Graphics 36 (2017) 1-16.

[73] A. J. Macleod, Acceleration of vector sequences by multi-dimensional delta-square methods, Communications in Applied Numerical Methods 2 (1986) 385-392.

[74] D. G. M. Anderson, Iterative procedures for nonlinear integral equations, J. ACM 12 (1965) $547-560$.

[75] C. Brezinski, M. Redivo-Zaglia, Y. Saad, Shanks sequence transformations and anderson acceleration, SIAM Review 60 (2018) 646-669. 